\title{
Radiatively scotogenic type-II seesaw and a relevant phenomenological analysis
}

\author{
Chuan-Hung Chen ${ }^{a}$ and Takaaki Nomura ${ }^{b}$ \\ ${ }^{a}$ Department of Physics, National Cheng-Kung University, \\ Tainan 70101, Taiwan \\ ${ }^{b}$ School of Physics, KIAS, \\ Seoul 02455, Korea \\ E-mail: physchen@mail.ncku.edu.tw, nomura@kias.re.kr
}

ABSTRACT: When a small vacuum expectation value of Higgs triplet $\left(v_{\Delta}\right)$ in the type-II seesaw model is required to explain neutrino oscillation data, a fine-tuning issue occurs on the mass-dimension lepton-number-violation (LNV) scalar coupling. Using the scotogenic approach, we investigate how a small LNV term is arisen through a radiative correction when an $Z_{2}$-odd vector-like lepton $(X)$ and an $Z_{2}$-odd right-handed Majorana lepton $(N)$ are introduced to the type-II seesaw model. Due to the dark matter (DM) direct detection constraints, the available DM candidate is the right-handed Majorana particle, whose mass depends on and is close to the $m_{X}$ parameter. Combing the constraints from the DM measurements, the $h \rightarrow \gamma \gamma$ decay, and the oblique $T$-parameter, it is found that the preferred range of $v_{\Delta}$ is approximately in the region of $10^{-5}-10^{-4} \mathrm{GeV}$; the mass difference between the doubly and the singly charged Higgs is less than $50 \mathrm{GeV}$, and the influence on the $h \rightarrow Z \gamma$ decay is not significant. Using the constrained parameters, we analyze the decays of each Higgs triplet scalar in detail, including the possible three-body decays when the kinematic condition is allowed. It is found that with the exception of doubly charged Higgs, scalar mixing effects play an important role in the Higgs triplet two-body decays when the scalar masses are near-degenerate. In the non-degenerate mass region, the branching ratios of the Higgs triplet decays are dominated by the three-body decays.

Keywords: Beyond Standard Model, Higgs Physics, Neutrino Physics

ARXIV EPRINT: 1906.10516 


\section{Contents}

1 Introduction 1

2 The model 3

2.1 Heavy Majorana masses 4

2.2 Gauge couplings of $Z_{2}$-odd particles 5

3 Scalar potential and Yukawa sector $\quad 6$

3.1 Scalar mass spectra and scalar couplings $\quad 8$

3.2 Yukawa couplings and neutrino masses 9

4 The constraints $\quad 10$

$\begin{array}{lll}\text { 4.1 Constraint from the neutrino data } & 10\end{array}$

4.2 Constraints from the DM relic density and the DM direct detections 11

$\begin{array}{lll}4.3 & \text { T-parameter and } h \rightarrow \gamma \gamma \text { constraints } & 16\end{array}$

5 Phenomenological analysis $\quad 18$

$\begin{array}{lll}5.1 & \text { Signal strength for } h \rightarrow Z \gamma & 18\end{array}$

$\begin{array}{lll}5.2 & \text { Doubly charged Higgs decays } & 19\end{array}$

5.3 Singly charged Higgs decays 21

$5.4 H^{0}$ and $A^{0}$ decays 22

6 Conclusion $\quad 24$

$\begin{array}{lr}\text { A Scalar mass squares and mixing angles } & 26\end{array}$

$\begin{array}{lr}\text { B Higgs triplet gauge coupling } & 27\end{array}$

$\begin{array}{ll}\text { C Loop integral functions } & 28\end{array}$

\section{Introduction}

An extension of the standard model (SM) is necessary due to the observed massive neutrinos. If the origin of neutrino masses arises from a similar Brout-Englert-Higgs mechanism in the SM [1-3], where the $W^{ \pm}$and $Z$ gauge bosons, the quarks, and the charged leptons obtain their masses through a Higgs doublet $(H)$, it is natural to introduce a Higgs triplet $(\Delta)$ to the SM as a neutrino mass source. Hereafter, we call the Higgs triplet model the type-II seesaw model [4-8]. Since only the left-handed leptons couple to the Higgs triplet, neutrinos are the Majorana particles.

In addition to the Yukawa couplings, the neutrino masses are associated with the vacuum expectation value (VEV) of the Higgs triplet. In the minimal type-II seesaw model, 
it is known that the $\Delta \mathrm{VEV}$ indeed is dictated by the lepton-number softly breaking term $\mu_{\Delta} H^{T} i \tau_{2} \Delta^{\dagger} H$, which appears in the scalar potential. Thus, a fine-tuning issue on $\mu_{\Delta}$ is caused when the condition of $\mu_{D} \ll O\left(m_{W}\right)$ is required to explain the neutrino mass [9-11].

From the astrophysical observation, dark matter (DM) is introduced to explain more than $80 \%$ of non-baryonic matter. If DM is a kind of weakly interacting massive particle (WIMP), a radiatively scotogenic mechanism for generating the neutrino masses can be applied $[12,13]$, where the particles in the dark sector are the mediators in the loop Feynman diagrams. Various applications of scotogentic models can be found in [14-38].

In order to naturally obtain a small $\mu_{D}$ parameter in the type-II seesaw model, in this study, we consider that $\mu_{\Delta} H^{T} i \tau_{2} \Delta^{\dagger} H$ is suppressed at the tree level due to the leptonnumber symmetry; then, the necessary $\mu_{\Delta}$ term is radiatively induced through the scotogenic mechanism [39-41]. Since the minimal type-II seesaw model does not include any particles that belong to the invisible side, we inevitably have to add new dark representations to the type-II seesaw model. Because the Higgs triplet cannot couple to singlet fermions, the minimum representation that directly couples to the Higgs triplet is the $\mathrm{SU}(2)_{L}$ doublet fermion $(X)$. Due to $H$ and $X$ being the $\mathrm{SU}(2)_{L}$ doublets, in order to form a gauge invariant interaction, we can add one more singlet fermion $(N)$ into the model such that the $H, X$, and $N$ coupling can generate the $\mu_{\Delta}$ term through the one-loop level.

If the new representation set is assumed to be a minimal choice, due to the gauge anomaly free condition, the new doublet fermion can be a vector-like lepton doublet, and the singlet fermion can be a right-handed Majorana lepton without carrying any SM gauge quantum numbers. In addition, to have a stable DM candidate, we impose a $Z_{2}$-symmetry to the vector-like lepton doublet and right-handed singlet; that is, $X$ and $N$ belong to the dark representations. Thus, the loop-induced $\mu_{\Delta}$ term indeed arises from the leptonnumber soft breaking effects in the invisible sector.

The main characteristics in the simple extension of the type-II seesaw model can be summarized as follows: (a) The Dirac-type neutral component of $X$, denoted by $X^{0}$, becomes a Majorana-type lepton when the mixing with $N$ from the $X H N$ coupling occurs after electroweak symmetry breaking (EWSB); (b) the spin-independent (SI) and the spindependent (SD) DM-nucleon scatterings arise from the mediation of the $Z$ boson and the SM Higgs, respectively; (c) although the $X^{0}$ - and $N$-DM candidates can produce the observed DM relic density, the $X^{0}$ candidate is excluded by the constraints of the DM direct detection experiments; therefore, the DM candidate in this study is dominated by the Majorana particle $N$; (d) the loop-induced VEV of $\Delta$ can be in the range of $10^{-5}-10^{-4} \mathrm{GeV}$, whereas the Higgs triplet Yukawa couplings constrained by the neutrino oscillation data are in the range of $10^{-8}-10^{-7}$, and (e) the doubly charged Higgs $\left(H^{ \pm \pm}\right)$ favors decaying to the same sign $W$-boson and lepton pairs when $H^{ \pm \pm}$is as heavy as $m_{H^{ \pm \pm}} \sim 400$ and $800 \mathrm{GeV}$, respectively. In addition, we analyze the constraints from the Higgs diphoton decay and the oblique $T$ parameter [42]; as a result, $\left|m_{H^{ \pm \pm}}-m_{H^{ \pm}}\right| \lesssim$ $50 \mathrm{GeV}$ is allowed and the new physics influence on the $h \rightarrow Z \gamma$ decay is not significant.

In addition to the DM candidate and the origin of the neutrino masses, similar to the conventional type-II seesaw model, it is of interest to explore and probe the new scalars of the Higgs triplet at the LHC, especially the search for $H^{ \pm \pm}$. With an integrated luminosity 
of $12.9 \mathrm{fb}^{-1}$ at $\sqrt{s}=13 \mathrm{TeV}$, CMS reports that the bounds on $m_{H^{ \pm \pm}}$through the $\ell^{ \pm} \ell^{ \pm}(\ell=$ $e, \mu), \ell^{ \pm} \tau^{ \pm}$, and $\tau^{ \pm} \tau^{ \pm}$channels are between 800 and $820 \mathrm{GeV}$, between 643 and $714 \mathrm{GeV}$, and $535 \mathrm{GeV}$, respectively, where $B R\left(H^{++} \rightarrow \ell^{+} \ell^{\prime+}\right)=100 \%\left(\ell^{\prime}=e, \mu, \tau\right)$ for each lepton pair is used [43]. Using $36 \mathrm{fb}^{-1}$ of the integrated luminosity at $\sqrt{s}=13 \mathrm{TeV}$ and the same sign dilepton channels, ATLAS obtains the $m_{H^{ \pm \pm}}$lower bound from 770 to $870 \mathrm{GeV}$ with $B R\left(H^{++} \rightarrow \ell^{+} \ell^{+}\right)=100 \%$. Moreover, the $m_{H^{ \pm \pm}}$lower bound via the $H^{++} \rightarrow W^{+} W^{+}$ channel measured by ATLAS is given to be between 200 and $220 \mathrm{GeV}[45,46]$.

Based on the lower bound measurements of $m_{H^{ \pm \pm}}$, since the preferred $X$ mass in this study is close to $1 \mathrm{TeV}, H^{ \pm \pm}$decaying to the same sign charged heavy $X^{ \pm}$lepton pair is kinematically suppressed. Thus, the possible decay channels of the Higgs triplet are similar to the those of the conventional type-II seesaw model. Nevertheless, since the $\mu_{\Delta}$ parameter is dynamically generated in the model and mainly depends on the $X H N$ coupling, which is determined by the observed DM relic density and the DM direct detection experiments, the allowed $\triangle \mathrm{VEV}$ is limited in the narrow region of $10^{-5}-10^{-4} \mathrm{GeV}$, so, the Higgs triplet decay patterns are strongly correlated with the scalar couplings $\lambda_{1} H^{\dagger} H \operatorname{Tr}\left(\Delta^{\dagger} \Delta\right)$ and $\lambda_{4} H^{\dagger} \Delta \Delta^{\dagger} H$, where the $\lambda_{4}$ sign determines the mass ordering of the Higgs triplet scalars. Because the doubly charged Higgs search in the LHC has been broadly studied in the literature [47-68], we thus focus the analysis on the decays of each Higgs triplet scalar in detail.

The paper is organized as follows: in section 2, we discuss the extension of the SM, including the derivations of heavy $Z_{2}$-odd particle mixing and their gauge couplings. In addition to the loop-induced $\mu_{\Delta}$ term, we show all scalar mass spectra and the associated scalar mixings, the Higgs-triplet Yukawa couplings, and neutrino mass in section 3. In section 3, we study the possible constraints, such as neutrino data, DM relic density and DM direct detections, the oblique $T$ parameter, and $h \rightarrow \gamma \gamma$. We discuss the influence on $h \rightarrow Z \gamma$ and show the decays of each Higgs triplet in section 5. A conclusion is given in section 6 .

\section{The model}

In addition to the SM particles, we add one Higgs triplet $\Delta$, one vector-like lepton doublet $X_{R, L}$, and one $\mathrm{SU}(2)$ singlet heavy neutrino into the $\mathrm{SM}$, where their representations in $\mathrm{SU}(2)_{L} \times \mathrm{U}(1)_{Y}$ are given in table 1 . In order to avoid the Dirac neutrino mass term, we require that $X$ and $N$ are $Z_{2}$-odd states and that the others are $Z_{2}$-even; therefore, the lightest neutral particles of $X$ and $N$ could be the DM candidate. In addition, in order to dynamically generate the finite dimension-3 lepton-number violating term in the scalar potential, we assign that $X_{L(R)}, N$ and $\Delta$ carry the lepton numbers as $0(1), 0$ and 2 , respectively, where the lepton number symmetry is softly broken by the $X$ Dirac mass term. The detailed charge assignments of the introduced particles are shown in table 1.

Based on the chosen representations and charge assignments, the gauge invariant Yukawa couplings can be written as:

$$
\begin{aligned}
-\mathcal{L}_{Y}= & \bar{L} \mathbf{y}^{\ell} H \ell_{R}+L^{T} C i \tau_{2} \Delta \mathbf{y}_{\Delta}^{\ell} L+y_{R} X_{R}^{T} C i \tau_{2} \Delta X_{R} \\
& +y_{X} \bar{X}_{L} \tilde{H} N+\frac{m_{N}}{2} N^{T} C N+m_{X} \bar{X}_{L} X_{R}+H . c .
\end{aligned}
$$




\begin{tabular}{|c|c|c|c|}
\hline Particle & $\mathrm{SU}(2)_{L} \times \mathrm{U}(1)_{Y}$ & $Z_{2}$ & Lepton \# \\
\hline$X_{L}$ & $(2,-1)$ & -1 & 0 \\
\hline$X_{R}$ & $(2,-1)$ & -1 & 1 \\
\hline$N$ & $(1,0)$ & -1 & 0 \\
\hline$\Delta$ & $(3,2)$ & +1 & -2 \\
\hline
\end{tabular}

Table 1. Representations and charge assignments of the introduced particles.

where the flavor indices are suppressed; $C=i \gamma^{2} \gamma^{0}$ is charge conjugation matrix; $H$ is the SM Higgs doublet, $\tilde{H}=i \tau_{2} H^{*}, \tau_{2}$ is the Pauli matrix, and $L^{T}=(\nu, \ell)$ is the SM lepton doublet. It can be seen that the lepton number symmetry is explicitly broken by the $m_{X}$ dimension-3 terms. The Higgs doublet, vector-like lepton doublet, and Higgs triplet are respectively expressed as:

$$
\begin{aligned}
& H=\left(\begin{array}{c}
G^{+} \\
\Phi^{0}
\end{array}\right), X=\left(\begin{array}{c}
X^{0} \\
X^{-}
\end{array}\right), \\
& \Delta=\left(\begin{array}{cc}
\delta^{+} / \sqrt{2} & \delta^{++} \\
\Delta^{0} & -\delta^{+} / \sqrt{2}
\end{array}\right),
\end{aligned}
$$

with $\Phi^{0}=\left(v_{h}+\operatorname{Re}\left(\Phi^{0}\right)+i \operatorname{Im}\left(\Phi^{0}\right)\right) / \sqrt{2}$ and $\Delta^{0}=\left(v_{\Delta}+\operatorname{Re}\left(\Delta^{0}\right)+i \operatorname{Im}\left(\Delta^{0}\right)\right) / \sqrt{2}$, in which $v_{h}$ and $v_{\Delta}$ are the VEVs of the $\Phi^{0}$ and $\Delta^{0}$ fields, respectively. The VEVs and scalar masses are determined by the scalar potential.

\subsection{Heavy Majorana masses}

Because of the $X_{L} H N$ and $X_{R} \Delta X_{R}$ couplings, it is found that the Dirac-type $X^{0}$ not only mixes with Majorana particle $N$ but also has a Majorana mass, which is related to $v_{\Delta} X_{R}^{T} C X_{R}$ when $\Delta^{0}$ obtains a VEV. Thus, using the basis of $\left(X_{R}, X_{L}^{C}, N\right)$, the Majoranatype heavy fermion mass matrix is written as:

$$
M_{M}=\left(\begin{array}{ccc}
m_{0} & m_{X} & 0 \\
m_{X} & 0 & y_{X} v_{h} / \sqrt{2} \\
0 & y_{X} v_{h} / \sqrt{2} & m_{N}
\end{array}\right),
$$

with $m_{0}=\sqrt{2} y_{R} v_{\Delta}$. Since $v_{\Delta}$ is induced from one-loop in this study, it is expected that $m_{0} \ll m_{N, X}$. It is found that the $M_{M}$ eigenvalues can be approximately expressed as follows: for $m_{N}>m_{X}$,

$$
m_{N_{1}} \approx m_{X}-e_{X},-m_{N_{2}} \approx\left(m_{X}+e_{\delta}\right), m_{N_{3}} \approx m_{N}+e_{N},
$$

where we use $N_{i}$ as the Majorana particle eigenstates, and $e_{N, X}$ and $e_{\delta}$ are obtained as:

$$
\begin{aligned}
e_{N} & =\frac{y_{X}^{2} v_{h}^{2}}{2 m_{N}}, \\
e_{X} & =m_{X}+\frac{e_{N}}{2}-\sqrt{\left(m_{X}+e_{N} / 2\right)^{2}-m_{X} e_{N}}, \\
e_{\delta} & =e_{N}-e_{X} .
\end{aligned}
$$


For $m_{N}<m_{X}$, they are:

$$
m_{N_{1}} \approx m_{X}+e_{X},-m_{N_{2}} \approx\left(m_{X}+e_{\delta}\right), m_{N_{3}} \approx m_{N}-e_{N}
$$

where the corresponding $e_{N, X}$ and $e_{\delta}$ are given as:

$$
\begin{aligned}
& e_{N}=\frac{2 m_{X}^{2}}{m_{N}+m_{X}}\left(-\left(1-\frac{m_{N}^{2}}{m_{X}^{2}}\right)+\sqrt{\left(1-\frac{m_{N}^{2}}{m_{X}^{2}}\right)^{2}+\left(1+\frac{m_{N}}{m_{X}}\right) \frac{y_{X}^{2} v_{h}^{2}}{m_{X}^{2}}}\right), \\
& e_{X}=\frac{1}{2}\left(1+\frac{m_{N}}{m_{X}}\right) e_{N}, e_{\delta}=\frac{1}{2}\left(1-\frac{m_{N}}{m_{X}}\right) e_{N} .
\end{aligned}
$$

Based on the obtained eigenvalues, the $3 \times 3$ orthogonal matrix elements $\left(O_{i j}\right)$, which transform the $\left(X_{R}, X_{L}^{C}, N\right)$ state to the $\left(N_{1}, N_{2}, N_{3}\right)$ state, can be formulated as:

$$
\begin{aligned}
& O_{11}=\mathcal{N}_{1}^{-1} \frac{m_{X}}{m_{N_{1}}-m_{0}}, \quad O_{12}=\frac{1}{\mathcal{N}_{1}}, \quad O_{13}=-\mathcal{N}_{1}^{-1} \frac{y_{X} v}{\sqrt{2}\left(m_{N}-m_{N_{1}}\right)}, \\
& O_{21}=-\mathcal{N}_{2}^{-1} \frac{m_{X}}{m_{0}-m_{N_{2}}}, \quad O_{22}=\frac{1}{\mathcal{N}_{2}}, \quad O_{23}=-\mathcal{N}_{2}^{-1} \frac{y_{X} v}{\sqrt{2}\left(m_{N}-m_{N_{2}}\right)}, \\
& O_{31}=\mathcal{N}_{3}^{-1} \frac{m_{X}}{m_{N_{3}}-m_{0}}, \quad O_{32}=\frac{1}{\mathcal{N}_{3}}, \quad O_{33}=-\mathcal{N}_{3}^{-1} \frac{y_{X} v_{h}}{\sqrt{2}\left(m_{N}-m_{N_{3}}\right)},
\end{aligned}
$$

where $\mathcal{N}_{i}^{2}=\sum_{k} O_{i k}^{2}$ are the normalization factors.

\subsection{Gauge couplings of $Z_{2}$-odd particles}

If we define the Majorana states $\chi_{i}$ as $\chi_{i}=N_{i}+N_{i}^{C}=\chi_{i}^{C}$, which satisfy $P_{R} \chi_{i}=N_{i}$ and $P_{L} \chi_{i}=N_{i}^{C}$, the charged current interactions of the heavy fermions can be expressed as:

$$
\mathcal{L}^{C C}=-\frac{g}{\sqrt{2}} O_{i 1} \bar{\chi}_{i} \gamma^{\mu} P_{R} X_{R}^{-} W_{\mu}^{+}-\frac{g}{\sqrt{2}} O_{i 2} \bar{\chi}_{i} \gamma^{\mu} P_{L} X_{L}^{-} W_{\mu}^{+}+\text {H.c. },
$$

where the mixing matrix elements $O_{i j}$ for the neutral $Z_{2}$-odd particles are included. The neutral current interactions of the $Z$-gauge boson and the photon with the $Z_{2}$-odd particles can be obtained as:

$$
\begin{aligned}
\mathcal{L}^{N C}= & -\frac{g c_{i j}^{Z}}{2 c_{W}} \bar{\chi}_{i} \gamma^{\mu} \frac{\gamma_{5}}{2} \chi_{j} Z_{\mu}+\frac{g c_{2 W}}{2 c_{W}} \overline{X^{-}} \gamma^{\mu} X^{-} Z_{\mu} \\
& -e Q_{X} \overline{X^{-}} \gamma^{\mu} X^{-} A_{\mu}
\end{aligned}
$$

where $c_{W}=\cos \theta_{W}$ and $c_{2 W}=\cos 2 \theta_{W}$ with Weinberg angle $\theta_{W} ; X^{-}$includes $X_{R}^{-}$and $X_{L}^{-}, Q_{X}=-1$ is the $X^{-}$electric charge, and $c_{i j}^{Z}$ show the FCNC effects and are defined as:

$$
c_{i j}^{Z}=\left(O \operatorname{diag}(1,-1,0) O^{T}\right)_{i j}=O_{i 1} O_{j 1}-O_{i 2} O_{j 2} .
$$

From eq. (2.10), it can be seen that the $Z$-boson coupling to the $Z_{2}$-odd particle is through axial-vector currents; therefore, it will lead to the SD DM-nucleon elastic scattering.

When $N_{1}\left(\chi_{1}\right)$ is the DM candidate, in order to satisfy the DM direct detection constraints, we must require $c_{11}^{Z}$ to be small enough. From eq. (2.8), if we drop the $m_{0}$ and 
$y_{X} v_{h} / \sqrt{2}$ effects, it can be seen that $c_{11}^{Z}=0$. However, the case leads to $m_{N_{1}}=m_{N_{2}}$ and $c_{12}^{Z}=1$, where the DM-nucleon scattering occurs through $\chi_{1 R} \chi_{2 R} Z$ coupling (or $X^{0} X^{0} Z$ coupling). Hence, in addition to the $c_{11}^{Z}$ magnitude, we have to take proper $m_{0}$ and $y_{X} v_{h} / \sqrt{2}$ in such a way that the mass splitting between $N_{1}$ and $N_{2(3)}$ is large enough, so that the DM scattering off the nucleon through $N_{1} N_{2,3} Z$ coupling can be kinematically suppressed. If we take $m_{N}>m_{X}$, the mass splitting between $N_{1}$ and $N_{2}$ can be found to be $\Delta m_{12}=e_{X}+e_{\delta} \approx e_{N}$, and the $c_{11}^{Z}$ coefficient can be expressed as:

$$
c_{11}^{Z} \approx \frac{2}{\mathcal{N}_{1}^{2}} \frac{e_{X}+m_{0}}{m_{X}} .
$$

If $N_{3}\left(\chi_{3}\right)$ is the DM candidate, because $c_{33}^{Z}$ is small, we will show that the SD DM-nucleon scattering cross section is under the current PICO-60 [70] and Xenon1T [71] upper limits.

\section{Scalar potential and Yukawa sector}

According to the convention in $[68,72]$, we write the gauge invariant scalar potential as:

$$
\begin{aligned}
V(H, \Delta)= & -\mu^{2} H^{\dagger} H+\frac{\lambda}{4}\left(H^{\dagger} H\right)^{2}+M_{\Delta}^{2} \operatorname{Tr}\left(\Delta^{\dagger} \Delta\right)+\lambda_{1}\left(H^{\dagger} H\right) \operatorname{Tr}\left(\Delta^{\dagger} \Delta\right) \\
& +\lambda_{2}\left(\operatorname{Tr}\left(\Delta^{\dagger} \Delta\right)\right)^{2}+\lambda_{3} \operatorname{Tr}\left(\Delta^{\dagger} \Delta\right)^{2}+\lambda_{4} H^{\dagger} \Delta \Delta^{\dagger} H,
\end{aligned}
$$

where we take $\mu^{2}, \lambda>0$ for the purpose of spontaneously breaking the electroweak gauge symmetry. It can be seen that due to the lepton-number conservation, the dimension-3 $H^{T} i \tau_{2} \Delta^{\dagger} H$ term is suppressed at the tree level. Without this term, the Higgs triplet cannot obtain a VEV and the SM neutrinos are still massless. In order to generate the finite dimension- 3 term, we require that the right-handed $Z_{2}$-odd lepton doublet only couples to the Higgs triplet by assigning proper lepton numbers to $X_{R}$ and $X_{L}$, which are shown in table. 1. Thus, the finite $H^{T} i \tau_{2} \Delta^{\dagger} H$ term can be dynamically generated through a fermion loop, in which the $m_{X}$ lepton number violating effect is involved. The associated Feynman diagram is shown in figure 1, where the cross symbols denote the mass insertions of the $N$ and $X$ leptons. Thus, the resulting dimension-3 term can be expressed as:

$$
V(H, \Delta)_{\operatorname{dim}-3}=\mu_{\Delta} H^{T} i \tau_{2} \Delta^{\dagger} H+H . c .,
$$

where the $\mu_{\Delta}$ coefficient is obtained as:

$$
\begin{aligned}
\mu_{\Delta} & =\frac{y_{X}^{2} y_{R} m_{N}}{8 \pi^{2}} I_{\Delta}\left(\frac{m_{X}^{2}}{m_{N}^{2}}\right), \\
I_{\Delta}(x) & =-\frac{x}{1-x}-\frac{x \ln x}{(1-x)^{2}} .
\end{aligned}
$$

For clarity, we show the contours of $\mu_{\Delta}$ as a function of $y_{X}$ and $y_{R}$ in figure 2(a), where $m_{X}=80 \mathrm{GeV}$ and $m_{N}=400 \mathrm{GeV}$ are used. Clearly, we can easily obtain $\mu_{\Delta}<10^{-2} \mathrm{GeV}$ without extremely fine-tuning the $y_{R}$ and $y_{X}$ parameters. For comparison, we make a contour plot with $m_{X}=800 \mathrm{GeV}$ and $m_{N}=700 \mathrm{GeV}$ in figure $2(\mathrm{~b})$. We will show that the 


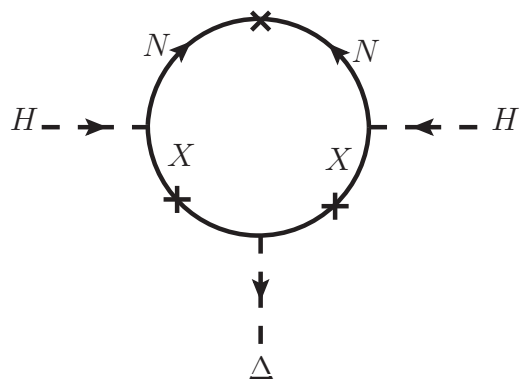

Figure 1. One-loop Feynman diagram for producing the $H^{T} i \tau_{2} \Delta^{\dagger} H$ term, where the cross symbols denote the mass insertions of the $N$ and $X$ leptons.
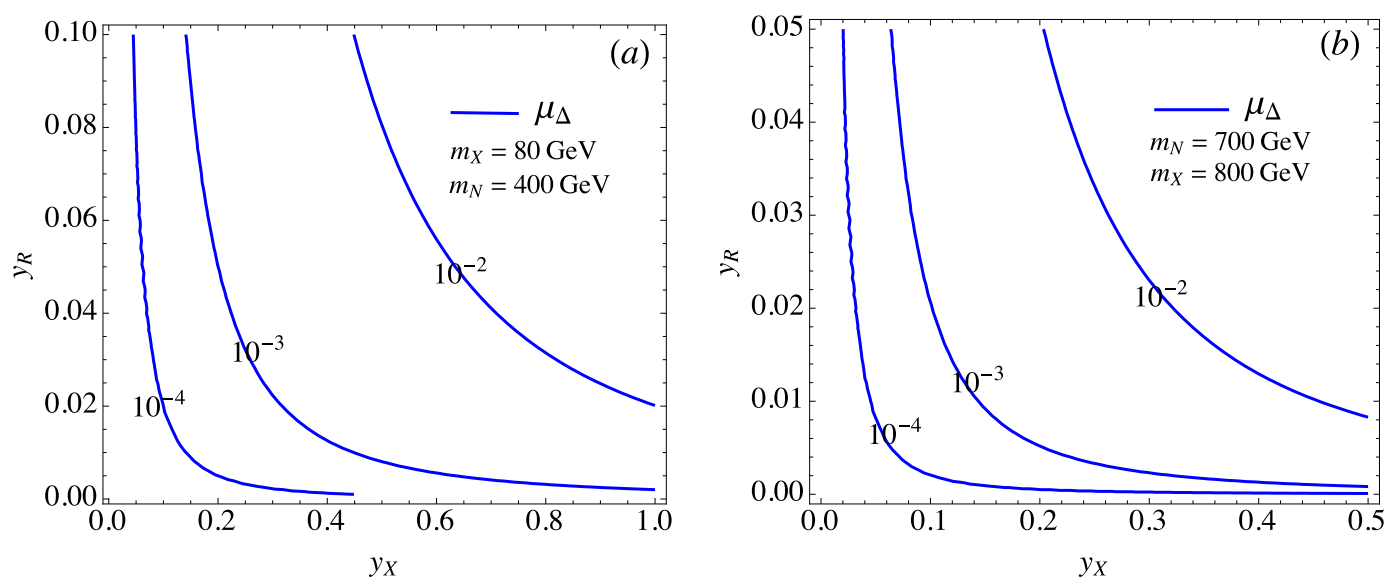

Figure 2. Contours of $\mu_{\Delta}$ as a function of $y_{X}$ and $y_{R}$ for (a) $\left(m_{X}, m_{N}\right)=(80,400) \mathrm{GeV}$ and (b) $\left(m_{X}, m_{N}\right)=(800,700) \mathrm{GeV}$.

former and latter plots correspond to the cases for which $\chi_{1}$ and $\chi_{3}$ are the DM candidates, respectively.

Combining eqs. (3.1) and (3.2), the minimum of the scalar potential can be obtained through $\partial V / \partial v_{h}=0$ and $\partial V / \partial v_{\Delta}=0$, and the minimum conditions can be written as:

$$
\begin{aligned}
-\mu^{2}+\frac{\lambda}{4} v_{h}^{2}+\frac{\lambda_{1}+\lambda_{4}}{2} v_{\Delta}^{2} & =\sqrt{2} \mu_{\Delta} v_{\Delta}, \\
\left(M_{\Delta}^{2}+\frac{\lambda_{1}+\lambda_{4}}{2} v_{h}^{2}+\left(\lambda_{2}+\lambda_{3}\right) v_{\Delta}^{2}\right) v_{\Delta} & =\frac{\mu_{\Delta} v_{h}^{2}}{\sqrt{2}} .
\end{aligned}
$$

Because we focus on the case of $\mu_{\Delta}<10^{-2} \mathrm{GeV}$, i.e., $v_{\Delta} \ll 1 \mathrm{GeV}$, when we neglect the small $\mu_{\Delta} v_{\Delta}$ and $v_{\Delta}^{2}$ effects, the VEVs of $\Phi^{0}$ and $\Delta^{0}$ can be respectively obtained as $v_{h} \approx \sqrt{4 \mu^{2} / \lambda}$ and

$$
v_{\Delta} \approx \frac{\mu_{\Delta} v_{h}^{2}}{\sqrt{2}\left[M_{\Delta}^{2}+v_{h}^{2}\left(\lambda_{1}+\lambda_{4}\right) / 2\right]}
$$

To obtain $v_{\Delta}>0$, we require $\mu_{\Delta}>0$, which is equivalent to $y_{R}>0$. Because of $v_{\Delta} \ll 1 \mathrm{GeV}$, the influence on the electroweak $\rho$-parameter can be neglected. We note that in addition to $\mu_{\Delta}$ and $M_{\Delta}, v_{\Delta}$ also depends on the $\lambda_{1,4}$ parameters. We will discuss the correlation between $v_{\Delta}$ and $\lambda_{1,4}$ when the constraints on the $\lambda_{1,4}$ parameters are studied. 
The vacuum stability of scalar potential has been studied in the literature $[72,77,78]$. Following the results in [72], the conditions for the scalar potential bounded from below in our notations can be written as:

$$
\begin{gathered}
\lambda>0, \quad \lambda_{2}+\lambda_{3}>0, \quad 2 \lambda_{2}+\lambda_{3}>0, \\
\lambda_{1}+\sqrt{\lambda\left(\lambda_{2}+\lambda_{3}\right)}>0, \quad \lambda_{1}+\lambda_{4}+\sqrt{\lambda\left(\lambda_{2}+\lambda_{3}\right)}>0,
\end{gathered}
$$

and

$$
\begin{array}{ll} 
& \left|\lambda_{4}\right| \sqrt{\lambda_{2}+\lambda_{3}}-\lambda_{3} \sqrt{2}>0, \\
\text { or } \quad & 2 \lambda_{1}+\lambda_{4}+\sqrt{\left(2 \lambda \lambda_{3}-\lambda_{4}^{2}\right)\left(2 \lambda_{2} / \lambda_{3}+1\right)}>0 .
\end{array}
$$

For the sake of satisfying perturbativity, we take $\lambda,\left|\lambda_{i}\right| \leq 4 \pi$ before we find the stricter constraints.

\subsection{Scalar mass spectra and scalar couplings}

In addition to the SM-like Higgs boson, the type-II seesaw model has two doubly and two singly charged Higgs, and one CP-even and one CP-odd scalar. The scalar mass spectra and the scalar-scalar couplings can be obtained from the scalar potential. Since the doubly charged Higgs does not mix with the other scalars, its mass can be easily obtained as:

$$
\begin{aligned}
m_{H^{ \pm \pm}}^{2} & =M_{\Delta}^{2}+\frac{\lambda_{2}}{2} v_{h}^{2}+\left(\lambda_{2}+\lambda_{3}\right) v_{\Delta}^{2} \\
& =\frac{\mu_{\Delta} v_{h}^{2}}{\sqrt{2} v_{\Delta}}-\frac{\lambda_{4}}{2} v_{h}^{2},
\end{aligned}
$$

where the minimal conditions in eq. (3.4) have been applied in the second line. The mass-square matrices for $\left(G^{-}, \Delta^{-}\right),\left(G^{0}, \operatorname{Im} \Delta^{0}\right)$, and $\left(\operatorname{Re} \Phi^{0}, \operatorname{Re} \Delta^{0}\right)$ can be respectively derived as:

$$
\begin{gathered}
\left(G^{-}, \Delta^{-}\right)\left(\begin{array}{cc}
\sqrt{2} v_{\Delta}\left(-\frac{\lambda_{4} v_{\Delta}}{2 \sqrt{2}}+\mu_{\Delta}\right) & -v_{h}\left(-\frac{\lambda_{4} v_{\Delta}}{2 \sqrt{2}}+\mu_{\Delta}\right) \\
-v_{h}\left(-\frac{\lambda_{4} v_{\Delta}}{2 \sqrt{2}}+\mu_{\Delta}\right) & \frac{v_{h}^{2}}{\sqrt{2} v_{\Delta}}\left(-\frac{\lambda_{4} v_{\Delta}}{2 \sqrt{2}}+\mu_{\Delta}\right)
\end{array}\right)\left(\begin{array}{c}
G^{+} \\
\Delta^{+}
\end{array}\right), \\
\frac{1}{2}\left(G^{0}, \operatorname{Im} \Delta^{0}\right)\left(\begin{array}{cc}
2 \sqrt{2} \mu_{\Delta} v_{\Delta} & -\sqrt{2} \mu_{\Delta} v_{h} \\
-\sqrt{2} \mu_{\Delta} v_{h} \mu_{\Delta} v_{h}^{2} /\left(\sqrt{2} v_{\Delta}\right)
\end{array}\right)\left(\begin{array}{c}
G^{0} \\
\operatorname{Im} \Delta^{0}
\end{array}\right), \\
\frac{1}{2}\left(\operatorname{Re} \Phi^{0}, \operatorname{Re} \Delta^{0}\right)\left(\begin{array}{cc}
\lambda v_{h}^{2} / 2 & \left(\lambda_{1}+\lambda_{4}\right) v_{h} v_{\Delta}-\sqrt{2} v_{h} \mu_{\Delta} \\
\left(\lambda_{1}+\lambda_{4}\right) v_{h} v_{\Delta}-\sqrt{2} v_{h} \mu_{\Delta} & \frac{\mu_{\Delta} v_{h}^{2}}{\sqrt{2} v_{\Delta}}+2 v_{\Delta}^{2}\left(\lambda_{2}+\lambda_{3}\right)
\end{array}\right)\left(\begin{array}{c}
\operatorname{Re} \Phi^{0} \\
\operatorname{Re} \Delta^{0}
\end{array}\right) .
\end{gathered}
$$

It can be easily verified that the determinants of the mass-square matrices in eqs. (3.9) and (3.10) vanish; that is, there exists a massless boson, which corresponds to the Goldstone boson, in each matrix. The detailed eigenvalues of the mass-square matrices and the associated mixing angles are shown in appendix A.

Because the off-diagonal elements in eq. (3.11) are much smaller than $v_{h}^{2} \mu_{\Delta} /\left(\sqrt{2} v_{\Delta}\right)$, the mixing effect between $\operatorname{Re} \Phi^{0}$ and $\operatorname{Re} \Delta^{0}$ can be approximately neglected if we only 
concentrate on the scalar spectrum. Thus, from the mass-square matrices, the mass squares for the physical bosons, such as the charged scalar $H^{ \pm}$, the CP-odd pseudoscalar $A^{0}$, and the two CP-even $H^{0}$ and $h$, can be written as:

$$
\begin{aligned}
m_{H^{ \pm}}^{2} & =\left(\frac{v_{h}^{2}}{\sqrt{2} v_{\Delta}}+\sqrt{2} v_{\Delta}\right)\left(-\frac{\lambda_{4} v_{\Delta}}{2 \sqrt{2}}+\mu_{\Delta}\right), \\
m_{A^{0}}^{2} & =\mu_{\Delta}\left(\frac{v_{h}^{2}}{\sqrt{2} v_{\Delta}}+2 \sqrt{2} v_{\Delta}\right), \\
m_{H^{0}}^{2} & \approx m_{A^{0}}^{2}-2 \sqrt{2} v_{\Delta} \mu_{\Delta}+2 v_{\Delta}^{2}\left(\lambda_{2}+\lambda_{3}\right),
\end{aligned}
$$

and $m_{h}^{2} \approx \lambda v_{h}^{2} / 2$, respectively, where $h$ is the SM-like Higgs boson. If we ignore the small $v_{\Delta}$ and $\mu_{\Delta}$ effects, it can be found that:

$$
\begin{aligned}
m_{H^{0}} \approx m_{A^{0}} & \approx \frac{v_{h}^{2} \mu_{\Delta}}{\sqrt{2} v_{\Delta}}, \\
m_{H^{ \pm \pm}}^{2}-m_{H^{ \pm}}^{2} & \approx-\frac{\lambda_{4} v_{h}^{2}}{4}, \\
m_{H^{ \pm}}^{2}-m_{H^{0}\left(A^{0}\right)}^{2} & \approx-\frac{\lambda_{4} v_{h}^{2}}{4},
\end{aligned}
$$

where the mass splittings in the Higgs triplet components can be constrained by the electroweak oblique parameters [42]. From eq. (3.13), we have the mass ordering $m_{H^{0}\left(A^{0}\right)}>$ $m_{H^{ \pm}}>m_{H^{ \pm \pm}}$when $\lambda_{4}>0$; however, the order is reversed when $\lambda_{4}<0$.

In order to study the Higgs precision measurement constraint, we write the Higgs trilinear couplings to the triplet scalars as:

$$
\begin{aligned}
-\mathcal{L}_{V} \supset & \lambda_{1} v_{h} h H^{--} H^{++}+\left(\lambda_{1}+\frac{\lambda_{4}}{2}\right) v_{h} h H^{-} H^{+} \\
+ & \frac{1}{2}\left(\lambda_{1}+\lambda_{4}\right) v_{h} h\left(H^{0} H^{0}+A^{0} A^{0}\right)+\frac{1}{2}\left(\left(\lambda_{1}+\lambda_{4}\right) v_{\Delta}-\sqrt{2} \mu_{\Delta}\right) h h H^{0} .
\end{aligned}
$$

The Higgs triplet couplings to the gauge bosons can be obtained from the kinetic terms, written as:

$$
\mathcal{L}_{\text {kin }}=\operatorname{Tr}\left[\left(D_{\mu} \Delta\right)^{\dagger}\left(D^{\mu} \Delta\right)\right]
$$

where the covariant derivative of the Higgs triplet is given as:

$$
D_{\mu} \Delta=\partial_{\mu} \Delta+i g\left[\frac{\boldsymbol{\tau}}{2} \cdot \mathbf{W}_{\mu}, \Delta\right]+i g^{\prime} B_{\mu} \Delta .
$$

The detailed trilinear couplings to gauge bosons can be found in appendix B.

\subsection{Yukawa couplings and neutrino masses}

Using the heavy Majorana flavor mixing matrix in eq. (2.8), the scalar Yukawa couplings to the heavy $Z_{2}$-odd fermions can be straightforwardly obtained as:

$$
\begin{aligned}
-\mathcal{L}_{Y}^{\text {odd }} \supset & \frac{1}{2}\left(\sqrt{2} y_{R} O_{i 1} O_{j 1}\right)\left(\bar{\chi}_{i} \chi_{j} H^{0}+i \bar{\chi}_{i} \gamma_{5} \chi_{j} A^{0}\right)+\frac{1}{2} \frac{y_{X} c_{i j}^{h}}{\sqrt{2}} \bar{\chi}_{i} \chi_{j} h \\
- & {\left[\sqrt{2} y_{R} O_{i 1} \bar{\chi}_{i} X_{R}^{-} H^{+}+\frac{1}{2}\left(2 y_{R}\right) X_{R}^{-T} C X_{R}^{-} H^{++}+H . c .\right], }
\end{aligned}
$$

with $c_{i j}^{h}=O_{i 2} O_{j 3}+O_{i 3} O_{j 2}$. 
In addition to the SM lepton coupling to the Higgs doublet, the SM left-handed leptons also couple to the Higgs triplet. When we derive the lepton couplings to the Higgs triplet in physical states, we have to simultaneously consider the $\mathbf{y}^{\ell}$ and $\mathbf{y}_{\Delta}^{\ell}$ terms in eq. (2.1). In terms of the components of the Higgs doublet and triplet, the relevant Yukawa couplings of $Z_{2}$-even leptons are written as:

$$
\begin{aligned}
&-\mathcal{L}_{Y}^{\text {even }} \supset \bar{\ell}_{L} \mathbf{y}^{\ell} \ell_{R} \frac{v+h}{\sqrt{2}}+\nu_{L}^{T} C \mathbf{y}_{\Delta}^{\ell} \nu_{L} \frac{v_{\Delta}+H^{0}+i A^{0}}{\sqrt{2}} \\
&-\sqrt{2} \nu^{T} \mathbf{y}_{\Delta}^{\ell} \ell_{L} H^{+}-\ell_{L}^{T} C \mathbf{y}_{\Delta}^{\ell} \ell_{L} H^{++}+H . c .
\end{aligned}
$$

where we have neglected the small $\mu_{\Delta}$ and $v_{\Delta}$ effects. To diagonalize the charged lepton and Majorana neutrino mass matrices, we introduce the unitary matrices for which the transformations are defined as: $\nu_{L} \rightarrow U_{\ell}^{\nu} \nu_{L}$ and $\ell_{L(R)} \rightarrow U_{L(R)}^{\ell} \ell_{L(R)}$. If we define $\mathbf{h}^{\ell} \equiv$ $U_{L}^{\ell *} \mathbf{y}_{\Delta}^{\ell} U_{L}^{\ell \dagger}$ and the Pontecorvo-Maki-Nakagawa-Sakata (PMNS) matrix as $U_{\mathrm{PMNS}}^{\dagger}=U_{L}^{\nu} U_{L}^{\ell \dagger}$, eq. (3.18) with respect to the lepton physical states can be written as:

$$
\begin{aligned}
-\mathcal{L}_{Y}^{\mathrm{even}} \supset & \bar{\ell}_{L} \mathbf{m}_{\ell}^{\text {dia }} \ell_{R}+\bar{\ell}_{L}\left(\frac{\mathbf{m}_{\ell}^{\text {dia }}}{v}\right) \ell_{R} h+\frac{1}{2} \nu_{L}^{T} C \mathbf{m}_{\nu}^{\text {dia }} \nu_{L}+\frac{1}{2} \nu_{L}^{T} C\left(\frac{\mathbf{m}_{\nu}^{\text {dia }}}{v_{\Delta}}\right) \nu_{L}\left(H^{0}+i A^{0}\right) \\
& -\sqrt{2} \nu^{T} C U_{\mathrm{PMNS}}^{T} \mathbf{h}^{\ell} \ell_{L} H^{+}-\frac{1}{2} \ell_{L}^{T} C\left(2 \mathbf{h}^{\ell}\right) \ell_{L} H^{++}+H . c .
\end{aligned}
$$

where the diagonal mass matrices are given as:

$$
\begin{aligned}
& \mathbf{m}_{\ell}^{\text {dia }}=\operatorname{diag}\left(m_{e}, m_{\mu}, m_{\tau}\right)=U_{L}^{\ell} \frac{\mathbf{y}^{\ell} v_{h}}{\sqrt{2}} U_{R}^{\ell \dagger}, \\
& \mathbf{m}_{\nu}^{\text {dia }}=\operatorname{diag}\left(m_{1}, m_{2}, m_{3}\right)=U_{\mathrm{PMNS}}^{T}\left(\sqrt{2} v_{\Delta} \mathbf{h}^{\ell}\right) U_{\mathrm{PMNS}} .
\end{aligned}
$$

In order to explain the neutrino data, it is necessary to have $v_{\Delta} \mathbf{h}^{\ell} \sim 10^{-2} \mathrm{eV}$. It will be shown that the partial decay widths of the Higgs triplet scalars decaying to leptons are sensitive to $v_{\Delta}$, which is dictated by the parameters, such as $M_{\Delta}, \lambda_{1}, \lambda_{4}$, and $\mu_{\Delta}$.

\section{The constraints}

In this section, we discuss the constraints, such as the neutrino mass data, the observed DM relic density, the DM direct detections, the T-parameter, and the Higgs to diphoton precision measurement. It will be found that the $\chi_{1}$-DM candidate will be excluded by the upper limits of the DM-nucleon scattering cross sections. Since the cross section upper limit of the SD DM-neutron scattering in Xenon1T [71] is smaller than that of the SD DM-proton scattering in PICO-60 [70], we take the Xenon1T data as the upper limit of the SD DM-nucleon scattering cross section and use it to bound the parameters.

\subsection{Constraint from the neutrino data}

From eq. (3.20), the matrix elements of $\mathbf{h}^{\ell}$ can be written as:

$$
\mathbf{h}_{i j}^{\ell}=\frac{1}{\sqrt{2} v_{\Delta}}\left(U_{\mathrm{PMNS}}^{*}\right)_{i k} m_{\nu k}\left(U_{\mathrm{PMNS}}^{*}\right)_{j k}
$$


where the sum in $k$ for all active light neutrinos is indicated. It can be seen that the $\mathbf{h}_{i j}^{\ell}$ magnitudes strongly depend on the $v_{\Delta}$ value. Using the PMNS matrix parametrized as [79]:

$$
\begin{aligned}
U_{\mathrm{PMNS}}= & \left(\begin{array}{ccc}
c_{12} c_{13} & s_{12} c_{13} & s_{13} e^{-i \delta} \\
-s_{12} c_{23}-c_{12} s_{23} s_{13} e^{i \delta} & c_{12} c_{23}-s_{12} s_{23} s_{13} e^{i \delta} & s_{23} c_{13} \\
s_{12} s_{23}-c_{12} c_{23} s_{13} e^{i \delta} & -c_{12} s_{23}-s_{12} c_{23} s_{13} e^{i \delta} & c_{23} c_{13}
\end{array}\right) \\
& \times \operatorname{diag}\left(1, e^{i \alpha_{21} / 2}, e^{i \alpha_{31} / 2}\right) \equiv U_{\nu} \times \operatorname{diag}\left(1, e^{i \alpha_{21} / 2}, e^{i \alpha_{31} / 2}\right)
\end{aligned}
$$

where $s_{i j} \equiv \sin \theta_{i j}, c_{i j} \equiv \cos \theta_{i j} ; \delta$ is the Dirac CP violating phase, and $\alpha_{21,31}$ are Majorana $\mathrm{CP}$ violating phases, and the experimental data through the neutrino oscillation measurements can be given as [79]:

$$
\begin{aligned}
\Delta m_{21}^{2} & =(7.53 \pm 0.18) \times 10^{-5} \mathrm{eV}^{2}, \sin ^{2} \theta_{12}=0.307 \pm 0.013 \\
\Delta m_{32}^{2} & =(2.51 \pm 0.05,-2.56 \pm 0.04) \times 10^{-3} \mathrm{eV}^{2}(\mathrm{NO}, \mathrm{IO}) \\
\sin ^{2} \theta_{23} & =\left(0.597_{-0.030}^{+0.024}, 0.592_{-0.030}^{+0.023}\right)(\mathrm{NO}, \mathrm{IO}) \\
\sin ^{2} \theta_{13} & =(2.12 \pm 0.08) \times 10^{-2}
\end{aligned}
$$

where $\Delta m_{i j}^{2} \equiv m_{i}^{2}-m_{j}^{2}$, and $\Delta m_{32}^{2}>0$ and $\Delta m_{32}^{2}<0$ denote the normal ordering (NO) and inverted ordering (IO), respectively. The uncertain sign in $m_{32}^{2}$ originates from the undetermined neutrino mass ordering. Since the neutrino oscillation experiments cannot detect the Majorana CP phases, for simplicity, we take $\alpha_{31,32}=0$ in the following numerical estimates.

According to the recent results obtained by a global fit analysis, the central values of $\theta_{i j}, \delta$, and $\Delta m_{i j}^{2}$ are given as [80]:

$$
\begin{array}{ll}
\text { NO }: & \theta_{12}=34.5^{\circ}, \quad \theta_{23}=47.7^{\circ}, \quad \theta_{13}=8.45^{\circ}, \quad \delta=218^{\circ}, \\
& \Delta m_{21}^{2}=7.55 \times 10^{-5} \mathrm{eV}^{2}, \quad \Delta m_{31}^{2}=2.50 \times 10^{-3} \mathrm{eV}^{2}, \\
\mathrm{IO}: & \theta_{12}=34.5^{\circ}, \quad \theta_{23}=47.9^{\circ}, \quad \theta_{13}=8.53^{\circ}, \quad \delta=281^{\circ}, \\
& \Delta m_{21}^{2}=7.55 \times 10^{-5} \mathrm{eV}^{2}, \quad \Delta m_{31}^{2}=-2.42 \times 10^{-3} \mathrm{eV}^{2},
\end{array}
$$

where $m_{1(3)}=0$ for NO (IO) is taken. Using these results, the corresponding $\mathbf{h}_{i j}^{\ell}$ Yukawa matrix element values are shown in table 2 , where the values are in units of $10^{-3} \mathrm{eV} /\left(2 v_{\Delta}\right)$. When $v_{\Delta}$ is fixed, the $\mathbf{h}_{i j}^{\ell}$ values then can be determined. With $v_{\Delta} \sim 10^{-4} \mathrm{GeV}$, it can be seen that the $\mathbf{h}_{i j}^{\ell}$ magnitudes can be in the range of $\sim(0.1,1) \times 10^{-7}$. Due to the small Yukawa couplings, it can be expected that the lepton-flavor violating effects will be small.

\subsection{Constraints from the DM relic density and the DM direct detections}

In this model, the DM candidate could be an $\chi_{1}$ or $\chi_{3}$ Majorana fermion. Regardless of which one is the DM candidate, it is necessary to examine that whether the involved couplings can produce the current correct DM relic abundance $\left(\Omega_{\mathrm{DM}} h^{2}\right)$, which is observed as in [86]:

$$
\Omega_{\mathrm{DM}}^{\mathrm{obs}} h^{2}=0.1199 \pm 0.0022 .
$$




\begin{tabular}{|c|cccccc|}
\hline & $\mathbf{h}_{11}^{\ell}$ & $\mathbf{h}_{12}^{\ell}$ & $\mathbf{h}_{13}^{\ell}$ & $\mathbf{h}_{22}^{\ell}$ & $\mathbf{h}_{23}^{\ell}$ & $\mathbf{h}_{33}^{\ell}$ \\
\hline $\mathrm{NO}\left(10^{-3} \mathrm{eV} / 2 v_{\Delta}\right)$ & $3.17 e^{i 0.34}$ & $3.73 e^{-i 1.93}$ & $7.33 e^{-i 2.69}$ & $29.91 e^{-i 0.013}$ & 21.38 & $24.93 e^{i 0.014}$ \\
\hline $\mathrm{IO}\left(10^{-3} \mathrm{eV} / 2 v_{\Delta}\right)$ & 47.60 & $5.26 e^{-i 1.72}$ & $4.84 e^{-i 1.81}$ & $21.44 e^{i 0.008}$ & $24.84 e^{i 3.13}$ & $26.51 e^{i 0.009}$ \\
\hline
\end{tabular}

Table 2. The $\mathbf{h}_{i j}^{\ell}$ Yukawa matrix element values (in units of $10^{-3} \mathrm{eV} / 2 v_{\Delta}$ ), where the central values obtained by a global fit analysis in [80] are applied.
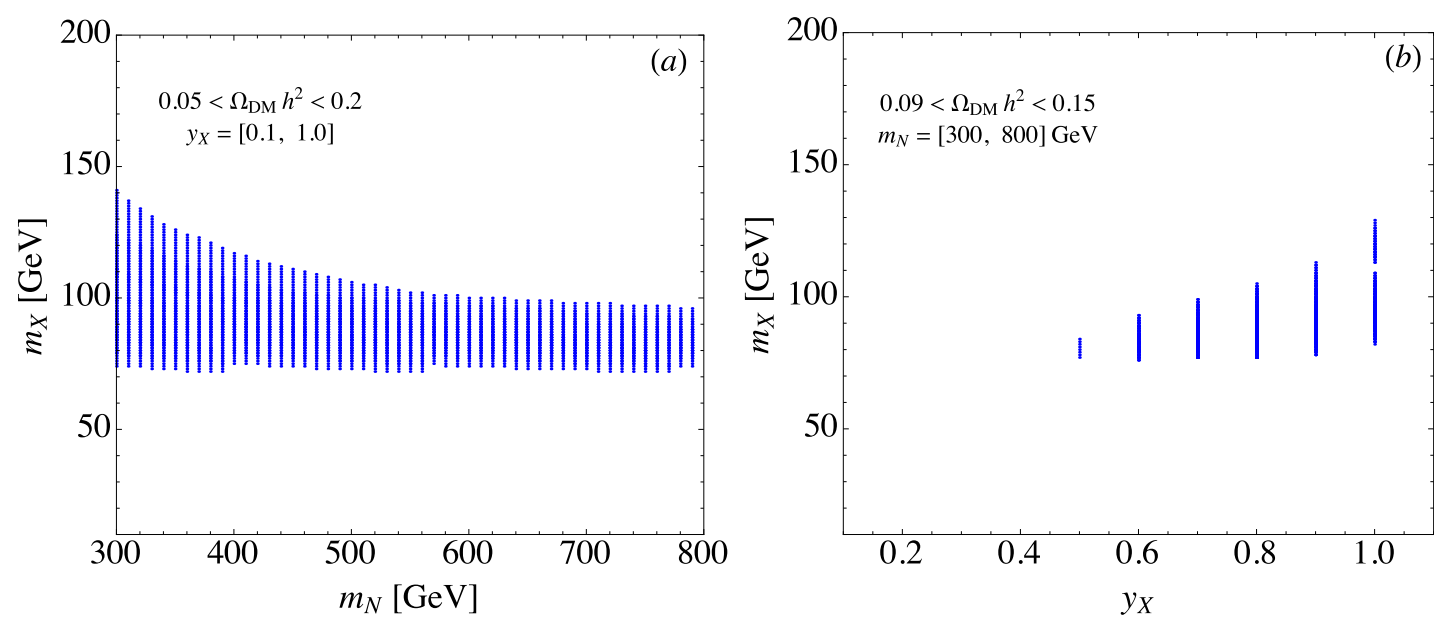

Figure 3. Allowed parameter space, which can produce the DM relic density in the region of $0.09<\Omega_{\mathrm{DM}} h^{2}<0.15$.

Since the DM relic density is inversely proportional to the product of the DM annihilation cross section and its velocity, i.e. $\langle\sigma v\rangle$, in addition to the thermal effects in the early time of the universe, we have to consider the DM annihilation and co-annihilation to the SM particles in the final states. In order to deal with the thermal effects and to calculate the $Z_{2}$-odd particle annihilation processes, we employ micrOmegas [75] with a choice of a unitary gauge. For clarity, we separately discuss the situations of $\chi_{1^{-}}$and $\chi_{3}$-DM in the following analysis. Although DM couples to the Higgs triplet, since we take the associated $y_{R}$ parameter to be $\lesssim O\left(10^{-2}\right)$, the effects indeed are small. Thus, we neglect the Higgs triplet contributions to the DM relic density.

When the DM candidate is the $\chi_{1}$ Majorana particle, because its origin is the $\mathrm{SU}(2)$ lepton doublet, and it has a large coupling to the SM gauge bosons, we require that the DM mass satisfies $m_{\chi_{1}}>45 \mathrm{GeV}$ due to the invisible $Z$ decay constraint. To avoid obtaining too large of a DM annihilation rate, the massive gauge boson pair production should be suppressed; that is, $\chi_{1}$ cannot be too heavy. In order to understand the correlation between $\Omega_{\mathrm{DM}} h^{2}$ and the $m_{N, X}$ and $y_{X}$ parameters, the scanned parameter regions are chosen as:

$$
m_{N}=[300,800] \mathrm{GeV}, m_{X}=[10,150] \mathrm{GeV}, y_{X}=[0.1,1.0],
$$

where we require that the resulting $\Omega_{\mathrm{DM}}$ satisfies $0.09<\Omega_{\mathrm{DM}} h^{2}<0.15$. We note that, in order to get more sampling points for illustration, the region of $\Omega_{\mathrm{DM}} h^{2}$ is taken slightly wider than the observed $\Omega_{\mathrm{DM}} h^{2}$. We show the allowed parameter space as a function of $m_{N}$ and $m_{X}$ and as a function of $y_{X}$ and $m_{X}$ in figure 3 (a) and (b), respectively. It can be seen that only $m_{X} \sim 90 \mathrm{GeV}$ and $y_{X}>0.5$ can fit the condition of $0.09<\Omega_{\mathrm{DM}} h^{2}<0.15$. Based 


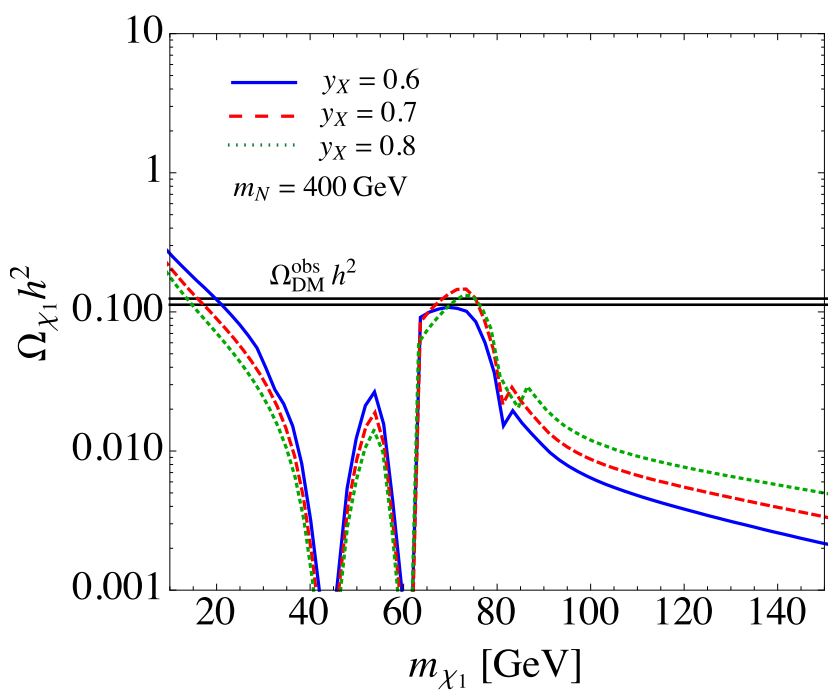

Figure 4. $\chi_{1}$-DM relic abundance as a function of $m_{\chi_{1}}$ for $y_{X}=0.6$ (solid), $y_{X}=0.7$ (dashed), and $y_{X}=0.8$ (dotted), where $m_{N}=400 \mathrm{GeV}$ is fixed, and the horizontal lines denote $\Omega_{\mathrm{DM}}^{\mathrm{obs}} h^{2}$ with $3 \sigma$ errors.

on the results, we show $\Omega_{\mathrm{DM}} h^{2}$ as a function of $m_{\chi_{1}}$ in figure 4 , where $m_{N}=400 \mathrm{GeV}$ is used, and the solid, dashed, and dotted lines denote the results of $y_{X}=0.6,0.7$, and 0.8 , respectively. Two dips denote $m_{\chi_{1}} \sim m_{Z} / 2$ and $m_{\chi_{1}} \sim m_{h} / 2$. It can be found that $m_{\chi_{1}} \sim$ $70 \mathrm{GeV}$ with $y_{X} \sim 0.7$ can fit the observed $\Omega_{\mathrm{DM}} h^{2}$ and can escape the constraint from the invisible $Z$ decay. Hence, without considering the DM direct detection constraints, the neutral component of the $Z_{2}$-odd lepton doublet could be the DM candidate in this model.

In addition to the DM relic density, we have to examine whether the same parameter space, which can fit $\Omega_{\mathrm{DM}}^{\mathrm{obs}} h^{2}$, is excluded by the DM direct detection experiments. In the model, it is found that the SI DM scattering off a nucleon is dictated by the Higgs mediation, whereas the SD scattering is through the $Z$-mediated effects. According to the interactions in eq. (2.10) and eq. (3.17), the relevant four-Fermi effective interactions for $\chi_{1}$ and the SM particles can be expressed as:

$$
\begin{aligned}
\mathcal{L}_{\chi N} \supset \frac{y_{X} c_{11}^{h}}{\sqrt{2} v m_{h}^{2}}\left(\bar{\chi}_{1} \chi_{1}\right) \sum_{q} m_{q} \bar{q} q-\frac{g c_{11}^{Z}}{2 c_{W} m_{Z}^{2}} \bar{\chi}_{1} \gamma^{\mu} \gamma_{5} \chi_{1} \sum_{q} \bar{q} \gamma_{\mu}\left(g_{V}^{q}+g_{A}^{q} \gamma_{5}\right) q, \\
g_{V}^{u}=\frac{g}{2 c_{W}}\left(\frac{1}{2}-\frac{4}{3} s_{W}^{2}\right), \quad g_{A}^{u}=\frac{1}{2} \\
g_{V}^{d}=\frac{g}{2 c_{W}}\left(-\frac{1}{2}+\frac{2}{3} s_{W}^{2}\right), \quad g_{A}^{d}=-\frac{1}{2}
\end{aligned}
$$

Accordingly, the $h$-mediated SI DM-nucleon scattering cross section can be written as [73]:

$$
\sigma_{h}^{S I}=\frac{y_{X}^{2}\left(c_{11}^{h}\right)^{2}}{8 \pi} \frac{m_{n}^{2} \mu_{\chi_{1} n}^{2} f_{N}^{2}}{v^{2} m_{h}^{4}}
$$

where $f_{N} \approx 0.3$, and $\mu_{\chi_{1} n}=m_{\chi_{1}} m_{n} /\left(m_{\chi_{1}}+m_{n}\right)$ is the DM-nucleon reduced mass. The 

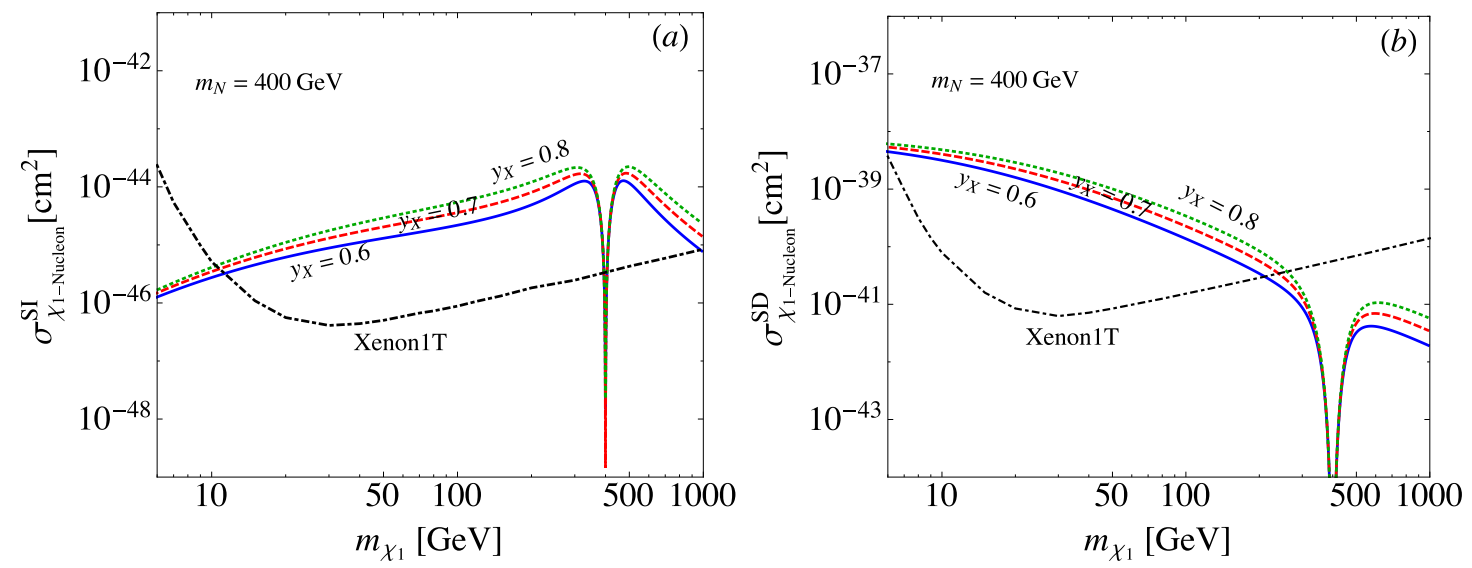

Figure 5. (a) $h$-mediated spin-independent and (b) $Z$-mediated spin-dependent DM-nucleon scattering cross sections as a function of $m_{\chi_{1}}$, where the solid, dashed, and dotted lines denote $y_{X}=0.6$, $y_{X}=0.7$, and $y_{X}=0.8$, respectively, and $m_{N}=400 \mathrm{GeV}$ is used. The dot-dashed lines in (a) and (b) are the Xenon1T results shown in [69, 71].

$Z$-mediated DM-nucleon scattering cross-section can be expressed as [74]

$$
\sigma_{Z}^{S D} \approx \frac{3 \mu_{\chi_{1} n}^{2}}{\pi}\left(\frac{g c_{11}^{Z}}{2 c_{W} m_{Z}^{2}}\right)^{2}\left[g_{A}^{u} \Delta_{u}^{n}+g_{A}^{d}\left(\Delta_{d}^{n}+\Delta_{s}^{n}\right)\right]
$$

where the quark spin fractions of the nucleon are taken as $\Delta_{u}^{n}=0.84, \Delta_{d}^{n}=-0.43$, and $\Delta_{s}^{n}=-0.08$ [75]. Using eq. (4.8) and eq. (4.9), we show $\sigma_{h}^{\mathrm{SI}}$ and $\sigma_{Z}^{\mathrm{SD}}$ as a function of $m_{\chi_{1}}$ in figure 5(a) and (b), respectively. A comparison with the results in figure 4 clearly shows that the allowed parameter regions, which can fit the observed $\Omega_{\mathrm{DM}} h^{2}$, are excluded by the current Xenon1T SI and SD measurements [69, 71]. Thus, it can be concluded that $\chi_{1}$ cannot be the DM candidate due to the strict constraints from the direct detection experiments.

Next, we discuss $\chi_{3}$ as the DM candidate. Since $\chi_{3}$ originates from an $\mathrm{SU}(2)$ singlet right-handed lepton, without the $y_{X}$ coupling, it can a heavy $Z_{2}$-odd sterile neutrino and doesn't couple to the SM particles. Therefore, the $\chi_{3}$ effects are all related to the $y_{X}$ parameter and the main interactions are through the Higgs couplings, i.e. the $\chi_{i} \chi_{3} h$ couplings shown in eq. (3.17). Similar to the $\chi_{1}$ case, to understand the correlation between $\Omega_{\mathrm{DM}} h^{2}$ and the $m_{N, X}$ and $y_{X}$ parameters, we choose the scanned parameter regions to be:

$$
m_{N}=[300,800] \mathrm{GeV}, m_{X}=[400,900] \mathrm{GeV}, y_{X}=[0.05,2.3],
$$

and the resulting $\Omega_{\mathrm{DM}} h^{2}$ is required to be in the region of $0.09<\Omega_{\mathrm{DM}} h^{2}<0.15$. As a result, the correlations between $m_{N}$ and $m_{X}$ and between $m_{N}$ and $y_{X}$ are shown in figure 6(a) and (b), respectively. From the plots, it can be seen that when $\chi_{3}$ is the DM candidate, the DM mass prefers to be heavy, and $y_{X}$ is of the order of 0.1 . In addition, according to the result shown in figure $6(\mathrm{a})$, it can be seen that the allowed maximum $m_{N}$ follows an approximate relation with $m_{X}$ as $m_{X}-m_{N} \sim 100 \mathrm{GeV}$. Based on the results, we show $\Omega_{\mathrm{DM}} h^{2}$ as a function of $m_{\chi_{3}}$ in figure 7 , where $m_{X}=800 \mathrm{GeV}$ is fixed, 

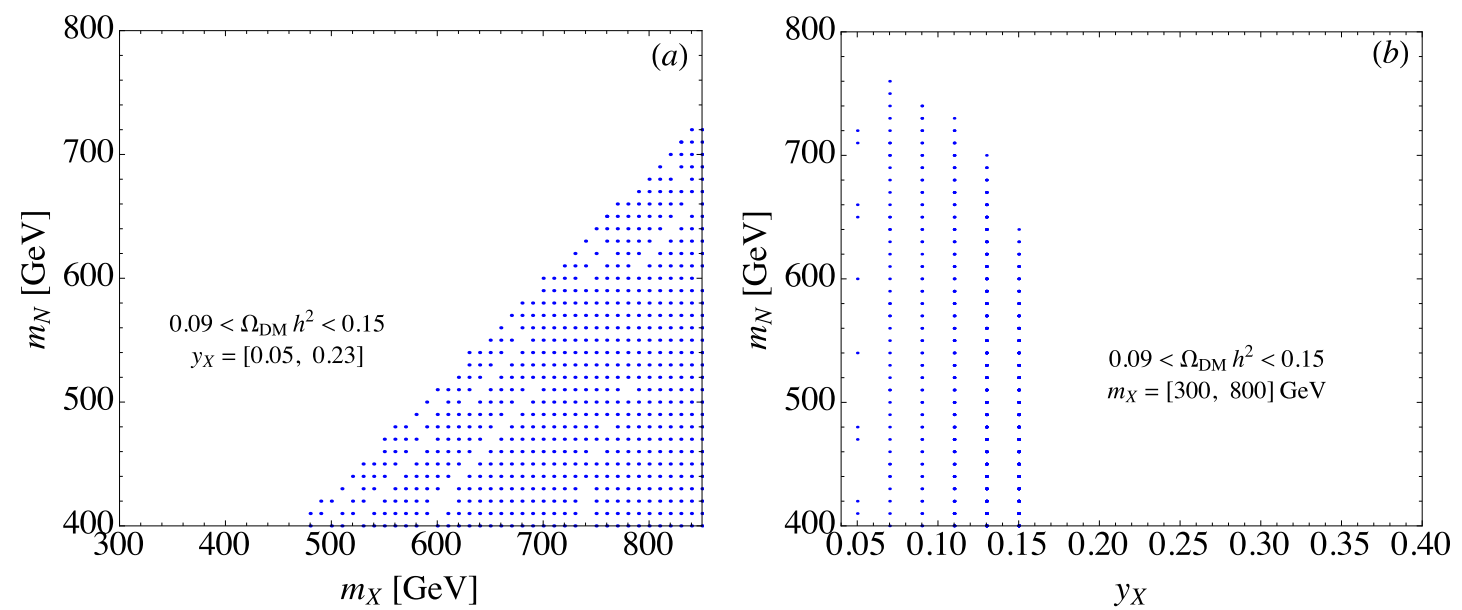

Figure 6. Legend is the same as in figure 3, but for the $m_{\chi_{3}}$ case.

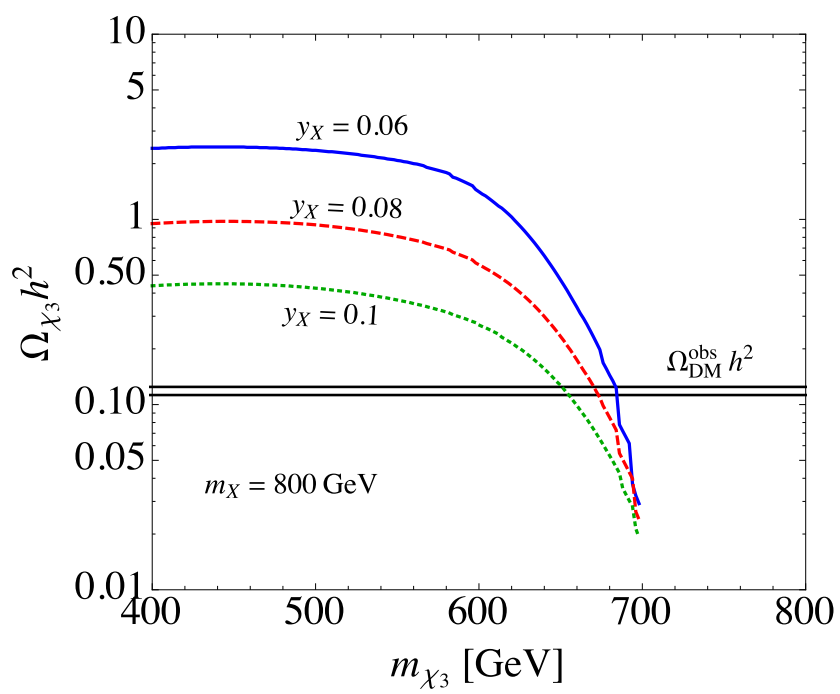

Figure 7. $\chi_{3}$-DM relic abundance for $y_{X}=0.06$ (solid), $y_{X}=0.08$ (dashed), and $y_{X}=0.10$ (dotted), where $m_{X}=800 \mathrm{GeV}$ is fixed, and the horizontal lines denote $\Omega_{\mathrm{DM}}^{\mathrm{obs}} h^{2}$ with $3 \sigma$ errors.

and the solid, dashed, and dotted lines denote the results of $y_{X}=0.06,0.08$, and 0.10 , respectively. It can be seen that $m_{\chi_{3}} \sim(680,670,650) \mathrm{GeV}$ with $y_{X} \sim(0.06,0.08,0.1)$ can fit the observed $\Omega_{\mathrm{DM}} h^{2}$. As mentioned earlier, the maximum of $m_{N}$ is close to $700 \mathrm{GeV}$ when $m_{X}=800 \mathrm{GeV}$ is taken; therefore, the three lines end at $m_{\chi_{3}} \approx 700 \mathrm{GeV}$. Due to $m_{\chi_{3}}>m_{Z, h}$, we can evade the constraints from the invisible $Z$ and $h$ decays.

Similar to the $\chi_{1}$ case, $\chi_{3}$ can contribute to the SI and SD DM-nucleon scatterings through the $h$ and $Z$ mediation, respectively. To estimate the elastic scattering cross sections, we can use the formulas in eqs. (4.8) and (4.9) by replacing $c_{11}^{h, Z}$ and $\mu_{\chi_{1} n}$ with $c_{33}^{h, Z}$ and $\mu_{\chi_{3} n}=m_{\chi_{3}} m_{n} /\left(m_{\chi_{3}}+m_{n}\right)$. Accordingly, we show the SI and SD $\chi_{3}$-nucleon scattering cross sections as a function of $m_{\chi_{3}}$ in figure $8(\mathrm{a})$ and (b), where $m_{X}=800 \mathrm{GeV}$ is used, and the solid, dashed, and dotted lines denote the results of $y_{X}=0.06,0.08$, and 0.1 , respectively. A comparison with the results shown in figure 7 reveals clearly that $\sigma_{h}^{S I}$ 

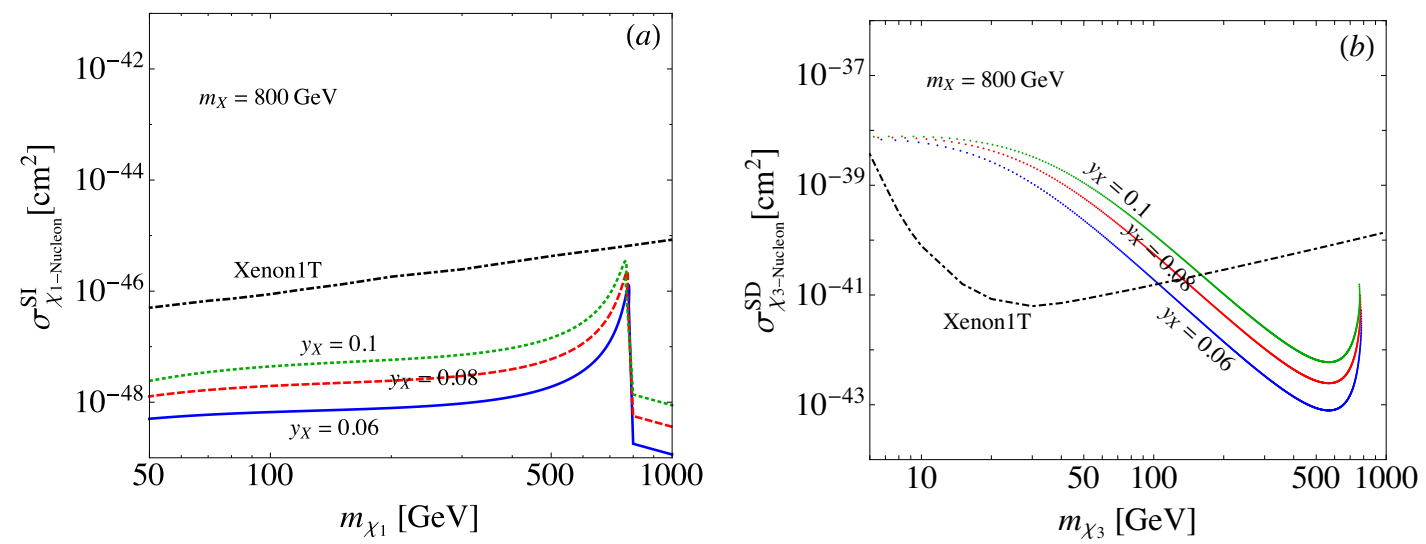

Figure 8. (a) $h$-mediated spin-independent and (b) $Z$-mediated spin-dependent DM-nucleon scattering cross sections, where the solid, dashed, and dotted lines denote $y_{X}=0.06, y_{X}=0.08$, and $y_{X}=0.1$, respectively, and $m_{X}=800 \mathrm{GeV}$ is used. The dot-dashed lines in (a) and (b) are the Xenon1T results shown in $[69,71]$.

and $\sigma_{Z}^{S D}$ at the $m_{\chi_{3}}$ value, which is determined by $\Omega_{\mathrm{DM}}^{\mathrm{obs}} h^{2}$, are all under the Xenon1T upper limits $[69,71]$. That is, the DM candidate in the model is the $\chi_{3} Z_{2}$-odd Majorana lepton. Note that a steep behavior in figure 8 (a) occurs when $m_{\chi_{3}}$ approaches $m_{X}=800 \mathrm{GeV}$, which is the upper limit of $m_{N}$.

\subsection{T-parameter and $h \rightarrow \gamma \gamma$ constraints}

From eq. (3.5), it can be seen that when $\mu_{\Delta}$ is fixed, $v_{\Delta}$ is determined by the $M_{\Delta}$ and $\lambda_{1,4}$ parameters. According to eq. (3.13), the mass ordering of the Higgs triplet bosons and their mass splittings are dictated by the $\lambda_{4}$ parameter. Moreover, the Higgs couplings to the doubly and singly charged Higgses also depend on $\lambda_{1,4}$. Thus, it can be expected that the electroweak oblique $T$ parameter [42] and the Higgs to diphoton precision measurement may give a strict constraint on the $\lambda_{1,4}$ parameters, where their values in principle could be $\left|\lambda_{1,4}\right| \leq 4 \pi$. Following the results obtained in [76], the $T$-parameter, which arises from the Higgs triplet, can be formulated as [76]:

$$
\begin{aligned}
T & =\frac{1}{8 \pi c_{W}^{2} s_{W}^{2}}\left[G\left(\frac{m_{H^{ \pm \pm}}^{2}}{m_{Z}^{2}}, \frac{m_{H^{ \pm}}^{2}}{m_{Z}^{2}}\right)+G\left(\frac{m_{H^{ \pm}}^{2}}{m_{Z}^{2}}, \frac{m_{H^{0}}^{2}}{m_{Z}^{2}}\right)\right], \\
G(x, y) & =x+y-\frac{2 x y}{x-y} \ln \frac{x}{y} .
\end{aligned}
$$

Basically, the mass splitting in the vector-like lepton doublet can also contribute to the T-parameter, where the mass difference is dictated by $e_{N}$. Using $y_{X}=0.1, m_{X}=800 \mathrm{GeV}$, and $m_{\chi_{3}}=700 \mathrm{GeV}$, we obtain $e_{N} \approx 3.2 \mathrm{GeV}$, where the resulting $T$ can be estimated to be $T \approx 0.8 \times 10^{-3}$ [38]. Since the influence on $T$-parameter is not significant, we drop the vector-like lepton doublet contribution in this study.

Next, we discuss the new physics contributions to $p p \rightarrow h \rightarrow \gamma \gamma$. As shown in appendix A, because the $h-H^{0}$ mixing angle is suppressed, the Higgs couplings to the SM quarks can be taken as unmodified. Thus, the $h$ production cross section in the $p p$ collisions 
is still from the SM contributions. Since the $h \rightarrow \gamma \gamma$ decay arises from the charged particle loops, in addition to the top and bottom quarks and the $W$-boson in the SM, the new physics effects in this model are from the doubly and singly charged Higgses. We note that although we have an $Z_{2}$-odd $X^{-}$in the model, the $h$ coupling to $X^{-}$has two suppression factors, where one is the $h-H^{0}$ mixing effect, and the other is the small $y_{R}$ parameter. Thus, we neglect the $X^{-}$contribution to $h \rightarrow \gamma \gamma$. Based on the results in [52, 81], we write the SM and Higgs triplet contributions to the partial decay width of $h \rightarrow \gamma \gamma$ as:

$$
\begin{aligned}
\Gamma(h \rightarrow \gamma \gamma) & =\frac{G_{F} \alpha^{2} m_{h}^{3}}{128 \sqrt{2} \pi^{3}}\left|\Gamma_{\gamma \gamma}^{\mathrm{SM}}+\Gamma_{\gamma \gamma}^{\Delta}\right|^{2}, \\
\Gamma_{\gamma \gamma}^{\Delta} & =\frac{\lambda_{1} v_{h}^{2}}{2 m_{H^{ \pm \pm}}^{2}} Q_{H^{ \pm \pm}}^{2} A_{0}\left(\frac{4 m_{H^{ \pm \pm}}^{2}}{m_{h}^{2}}\right)+\frac{\lambda_{1} v_{h}^{2}}{2 m_{H^{ \pm}}^{2}} Q_{H^{ \pm}}^{2} A_{0}\left(\frac{4 m_{H^{ \pm}}^{2}}{m_{h}^{2}}\right),
\end{aligned}
$$

where $\Gamma_{\gamma \gamma}^{\mathrm{SM}} \approx 6.50-i 0.02 ; Q_{H^{ \pm \pm}}=2$ and $Q_{H^{ \pm}}=1 ; A_{0}(\tau)=\tau(1-\tau f(\tau))$, and the loop function is defined as:

$$
f(x)= \begin{cases}\left(\sin ^{-1} \frac{1}{\sqrt{\tau}}\right)^{2}, & (\tau \geq 1), \\ -\frac{1}{4}\left(\ln \frac{1+\sqrt{1-\tau}}{1-\sqrt{1-\tau}}-i \pi\right)^{2}, & (\tau<1) .\end{cases}
$$

Thus, we can write the signal strength for $p p \rightarrow h \rightarrow \gamma \gamma$ as:

$$
\mu_{\gamma \gamma}=\frac{\sigma(p p \rightarrow h)}{\sigma(p p \rightarrow h)^{\mathrm{SM}}} \frac{B R(h \rightarrow \gamma \gamma)}{B R(h \rightarrow \gamma \gamma)^{\mathrm{SM}}} \approx \frac{B R(h \rightarrow \gamma \gamma)}{B R(h \rightarrow \gamma \gamma)^{\mathrm{SM}}}
$$

For numerical estimates, we take the Higgs width in the $\mathrm{SM}$ as $\Gamma^{\mathrm{SM}} \approx 4.07 \mathrm{MeV}$ [82]. The current Higgs to diphoton measurements from ATLAS and CMS at $\sqrt{s}=13 \mathrm{TeV}$ are given as $1.06 \pm 0.12$ [83] and $1.15 \pm 0.15$ [84], where the corresponding integrated luminosities are $79.8 \mathrm{fb}^{-1}$ and $77.4 \mathrm{fb}^{-1}$, respectively.

From eqs. (3.3) and (3.5), it is known that in addition to the $m_{X, N}$ and $y_{X, R}$ parameters, $v_{\Delta}$ also depends on the $\lambda_{1,4}$ constraints. Since the DM candidate in this model is $\chi_{3}$, and its mass is determined to be $m_{\chi_{3}} \sim 680 \mathrm{GeV}$ when $m_{X} \sim 800 \mathrm{GeV}$ is used, in order to simplify the study on the $\lambda_{1,4}$ constraints, we fix $m_{N(X)}=700(800) \mathrm{GeV}, y_{X}=0.1$, and $y_{R}=0.01$, where the corresponding $\mu_{\Delta}$ value is $4.8 \times 10^{-4} \mathrm{GeV}$. Using the introduced formulas for the $T$-parameter and $\mu_{\gamma \gamma}$, we show $T$-parameter, $\mu_{\gamma \gamma}, m_{H^{ \pm \pm}}-m_{H^{ \pm}}$, and $v_{\Delta}$ as a function of $\lambda_{1}$ and $\lambda_{4}$ in figure 9 , where the plots (a) and (b) correspond to $M_{\Delta}=400 \mathrm{GeV}$ and $M_{\Delta}=800 \mathrm{GeV}$, respectively.

From the resulting plots, we find: (a) Due to the $T$-parameter constraint, $\mid m_{H^{ \pm \pm}}-$ $m_{H^{ \pm}} \mid \lesssim 50 \mathrm{GeV}$, which is consistent with the results shown in $[61,85]$; (b) using the ATLAS result of $\mu_{\gamma \gamma}=1.06 \pm 0.12$, the $\lambda_{1}$ parameter is bounded to be $\lambda_{1}=(-0.8,2.63)$ and $\lambda_{1}=(-2.8,10.2)$ for $M_{\Delta}=400 \mathrm{GeV}$ and $M_{\Delta}=800 \mathrm{GeV}$, respectively, and (d) the allowed $v_{\Delta}$ range, which fits the $T$-parameter and $\mu_{\gamma \gamma}$ constraints, is obtained as: $v_{\Delta} \approx(0.63,2.6)[(0.185,0.48)] \times 10^{-4} \mathrm{GeV}$ for $M_{\Delta}=400[800] \mathrm{GeV}$. It can be seen that the allowed $\lambda_{1}$ is mostly in the region of $\lambda_{1}>0$, and the allowed $\lambda_{1}$ can reach a value 

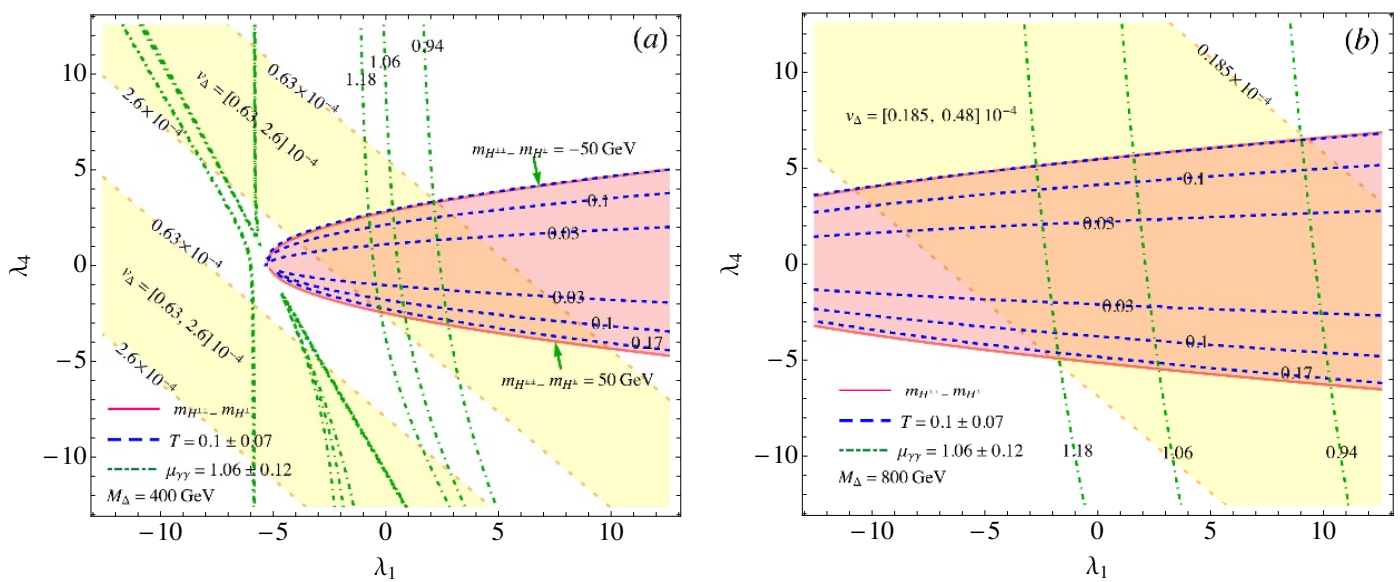

Figure 9. Constraints from the oblique $T$-parameter (dashed) and the $h \rightarrow \gamma \gamma$ (dot-dashed) precision measurement, where (a) [(b)] corresponds to the case with $M_{\Delta}=400[800] \mathrm{GeV}$ and $\mu_{\Delta}=4.8 \times 10^{-4} \mathrm{GeV}$. The area enclosed by the solid line denotes $m_{H^{ \pm \pm}}-m_{H^{ \pm}}=(-50,50) \mathrm{GeV}$. The $v_{\Delta}$ regions are $(0.63,2.6) \times 10^{-4} \mathrm{GeV}$ and $(0.185,0.48) \times 10^{-4} \mathrm{GeV}$ for $M_{\Delta}=400 \mathrm{GeV}$ and $M_{\Delta}=800 \mathrm{GeV}$, respectively.

of 10 when $M_{\Delta}$ approaches to $1 \mathrm{TeV}$. In addition, the $\lambda_{4}$ parameter is bounded in the region of $(1.1,3.4)$ and $(-2.78,-0.9)$ for $M_{\Delta}=400 \mathrm{GeV}$ and in the region of $(2.12,6.50)$ and $(-5.9,-2.0)$ for $M_{\Delta}=800 \mathrm{GeV}$. We note that the constraints cannot determine the sign of the $\lambda_{4}$ parameter; thus, the mass order, i.e. $m_{H^{0}\left(A^{0}\right)} \lesssim m_{H^{ \pm}} \lesssim m_{H^{ \pm \pm}}$or $m_{H^{ \pm \pm}} \lesssim m_{H^{ \pm}} \lesssim m_{H^{0}\left(A^{0}\right)}$, is still uncertain in the model.

\section{Phenomenological analysis}

After analyzing the potential constraints, in this section, we study the relevant phenomenology in detail, such as the $h \rightarrow Z \gamma$ and $H^{ \pm \pm}, H^{ \pm}$, and $H^{0}\left(A^{0}\right)$ decays. From the earlier analysis, since $m_{X}$ is taken to be $800 \mathrm{GeV}$, the processes, in which the Higgs triplet decays to the vector-like leptons, are kinematically suppressed when we focus on the study with $m_{\Delta}<1 \mathrm{TeV}$; therefore, we only consider the SM particles in the final states, where the three-body decays are also included when the kinematic condition is allowed. When the final states are all leptons, for simplicity, we sum up all possible lepton flavors. In addition, since the neutrino constraints from the NO and IO are similar in most lepton Yukawa couplings, hereafter, we only use the NO constraint as the inputs.

\subsection{Signal strength for $h \rightarrow Z \gamma$}

We have shown that the Higgs to diphoton measurement can bound the Higgs couplings to $H^{ \pm \pm}$and $H^{ \pm}$, which is dominated by the $\lambda_{1}$ parameter. Since the same couplings can also contribute to the loop-induced $h \rightarrow Z \gamma$, with the constrained parameters, we can predict the $h \rightarrow Z \gamma$ in the model. Thus, similar to the case in $h \rightarrow \gamma \gamma$, the signal strength of $h \rightarrow Z \gamma$ can be expressed as:

$$
\mu_{Z \gamma}=\frac{\sigma(p p \rightarrow h)}{\sigma(p p \rightarrow h)^{\mathrm{SM}}} \frac{B R(h \rightarrow Z \gamma)}{B R(h \rightarrow Z \gamma)^{\mathrm{SM}}} \approx \frac{B R(h \rightarrow Z \gamma)}{B R(h \rightarrow Z \gamma)^{\mathrm{SM}}},
$$



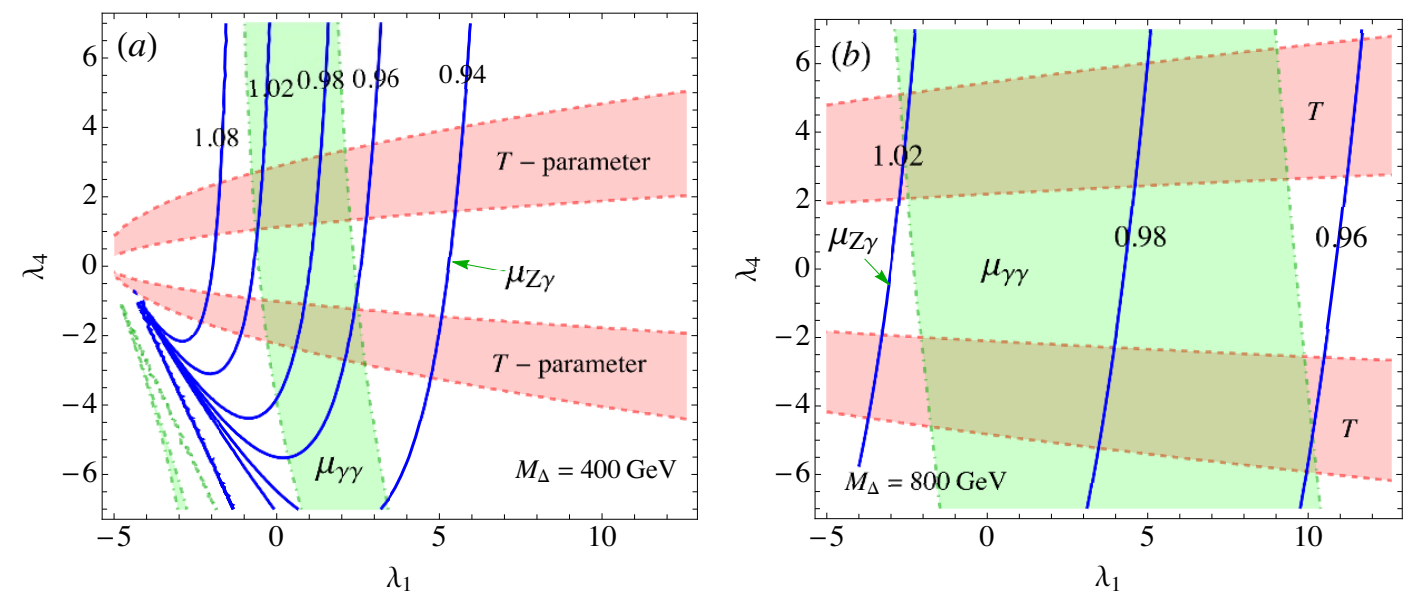

Figure 10. Contours for signal strength of $h \rightarrow Z \gamma$ as a function of $\lambda_{1}$ and $\lambda_{4}$ for (a) $M_{\Delta}=400 \mathrm{GeV}$ and (b) $M_{\Delta}=800 \mathrm{GeV}$, where the $T$-parameter and $\mu_{\gamma \gamma}$ constraints are also shown.

where the $h$ production cross section is dominated by the SM effects in the model, and the current upper limit is $\mu_{Z \gamma}<6.6$ [79].

Based on the results in $[57,81,87-90]$, we write the partial decay rate for $h \rightarrow Z \gamma$ as:

$$
\Gamma(h \rightarrow Z \gamma)=\frac{G_{F} \alpha m_{W}^{2} m_{h}^{3}}{64 \pi^{4}}\left(1-\frac{m_{Z}^{2}}{m_{h}^{2}}\right)^{3}\left|A_{\mathrm{SM}}+A_{\Delta}\right|^{2},
$$

where the SM and Higgs triplet contributions can be expressed as [81, 90]:

$$
\begin{aligned}
A_{\mathrm{SM}} & =-\frac{N_{C}}{c_{W}} \sum_{f} Q_{f}\left(2 I_{3}^{f}-4 Q_{f} s_{W}^{2}\right) A_{1 / 2}^{h}\left(\tau_{h}^{f}, \tau_{Z}^{f}\right)-c_{W} A_{1}^{h}\left(\tau_{h}^{W}, \tau_{Z}^{W}\right), \\
A_{\Delta} & =2 s W g_{Z 2 H^{ \pm}} g_{h 2 H^{ \pm}} A_{0}^{h}\left(\tau_{h}^{H^{ \pm}}, \tau_{Z}^{H^{ \pm}}\right)+4 s_{W} g_{Z 2 H^{ \pm \pm}} g_{h 2 H^{ \pm \pm}} A_{0}^{h}\left(\tau_{h}^{H^{ \pm \pm}}, \tau_{Z}^{H^{ \pm \pm}}\right) .
\end{aligned}
$$

Here, $N_{C}=3$ is the color number; $\tau_{h(Z)}^{i}=4 m_{i}^{2} / m_{h(Z)}^{2}, Q_{f}$ is the electric charge of $f$ fermion; $I_{3}^{f}$ is the third component of weak isospin of $f$ fermion, and the charged Higgs couplings to $h$ and $Z$ bosons are given as:

$$
\begin{aligned}
g_{h 2 H^{ \pm}} & =\frac{m_{W}}{g m_{H^{ \pm}}^{2}}\left(\lambda_{1}+\frac{\lambda_{4}}{2}\right) v_{h}, & g_{h 2 H^{ \pm \pm}} & =\frac{m_{W}}{g m_{H^{ \pm \pm}}^{2}} \lambda_{1} v_{h}, \\
g_{Z 2 H^{ \pm}} & =-\tan \theta_{W}, & g_{Z 2 H^{ \pm \pm}} & =2 \cot 2 \theta_{W} .
\end{aligned}
$$

The detailed loop functions $A_{0,1 / 2,1}^{h}$ can be found in appendix C. Accordingly, we show the $\mu_{Z \gamma}$ contours as a function of $\lambda_{1}$ and $\lambda_{4}$ in figure 10 (a) and (b) for $M_{\Delta}=400 \mathrm{GeV}$ and $M_{\Delta}=800 \mathrm{GeV}$, respectively, where the $T$-parameter and $\mu_{\gamma \gamma}$ constraints shown in figure 9 are included. From the plots, it can be seen that the influence from the Higgs-triplet charged particles is $\Delta \mu_{Z \gamma}=\left|\mu_{Z \gamma}^{\mathrm{SM}}-\mu_{Z \gamma}\right| \lesssim 4 \%$ and is not significant.

\subsection{Doubly charged Higgs decays}

The most peculiar phenomena in a type-II seesaw model should be the doubly chargedHiggs decays, where the final states in the decays are two singly charged particles. If 
$m_{H^{ \pm}}>m_{H^{ \pm \pm}}$, the final states are the same sign charged-lepton pair and $W$-boson pair; however, if $m_{H^{ \pm}}<m_{H^{ \pm \pm}}$, in addition to the leptons and the $W$-boson, we also have the three-body decays through the decay chain $H^{++} \rightarrow H^{+} W^{*} \rightarrow H^{+} \bar{f} f^{\prime}$, where $f\left(f^{\prime}\right)$ denotes the possible final states, and for simplicity, we take $f\left(f^{\prime}\right)$ to be massless. Although the $\mathrm{H}^{++} \rightarrow \mathrm{H}^{+*} \mathrm{~W}^{+}$decay is possible in principle, because the off-shell $\mathrm{H}^{+}$decays are associated with the small couplings, e.g. $v_{\Delta}$ and $\mathbf{h}_{i j}^{\ell}$, we neglect their contributions.

According to the introduced gauge and Yukawa couplings, the two-body $H^{ \pm \pm}$partial decay rates can be expressed as:

$$
\begin{aligned}
\Gamma\left(H^{++} \rightarrow W^{+} W^{+}\right) & =\frac{g^{4} v_{\Delta}^{2}}{16 \pi m_{H^{ \pm \pm}}}\left(2+\frac{\left(1-2 y_{W}\right)^{2}}{4 y_{W}^{2}}\right)\left(1-4 y_{W}\right)^{1 / 2}, \\
\Gamma\left(H^{++} \rightarrow \ell_{i}^{+} \ell_{j}^{+}\right) & =\frac{S_{i j}}{4 \pi}\left|\mathbf{h}_{i j}^{\ell}\right|^{2} m_{H^{ \pm \pm}},
\end{aligned}
$$

where $y_{W}=m_{W}^{2} / m_{H^{ \pm \pm}}^{2}, S_{i i}=1 / 2$, and $S_{i j}=1$ for $i \neq j$. For $\lambda_{4}<0, m_{H^{ \pm \pm}}$is the heaviest Higgs triplet; then, the three-body partial decay rate for $H^{++} \rightarrow H^{+} W^{+*}$ can be expressed as:

$$
\begin{aligned}
\Gamma\left(H^{++} \rightarrow H^{+} W^{+*}\right) & =\frac{3 g^{4} m_{H^{ \pm \pm}}}{2^{8} \pi^{3}} J_{0}\left(y_{W}, y_{H^{ \pm}}\right), \\
J_{0}\left(y_{W}, y_{H^{ \pm}}\right) & =\int_{s_{\min }}^{s_{\max }} \frac{\left(\left(1-y_{H^{ \pm}}+s\right)^{2}-4 s\right)^{3 / 2}}{\left(s-y_{W}\right)^{2}},
\end{aligned}
$$

with $y_{H^{ \pm}}=m_{H^{ \pm}}^{2} / m_{H^{ \pm \pm}}^{2}, s_{\min }=0$, and $s_{\max }=\left(1-\sqrt{y_{H^{ \pm}}}\right)^{2}$. The phase space integral can be simplified as:

$$
\begin{aligned}
J_{0}(a, b)= & \frac{1}{2 a}(1-b)\left(9 a(1+b)-2(1-b)^{2}-6 a^{2}\right)-3\left(1-2 a+(b-a)^{2}\right) \ln \sqrt{b} \\
& -3(1-a+b) \sqrt{-\lambda(a, b)}\left(\tan ^{-1} \frac{1-a-b}{\sqrt{-\lambda(a, b)}}+\tan ^{-1} \frac{1+a-b}{\sqrt{-\lambda(a, b)}}\right),
\end{aligned}
$$

with $\lambda(a, b)=1+a^{2}+b^{2}-2 a-2 b-2 a b$. If we assume that the main $H^{++}$decay modes are $W^{+} W^{+}, \ell_{i}^{+} \ell_{j}^{+}$, and $H^{+} W^{+*}$, the relative BRs as a function of $\lambda_{4}$ can be shown in figure 11 (a) and (b), where $M_{\Delta}=400 \mathrm{GeV}$ and $\lambda_{1}=2.5$ are used in plot (a) and $M_{\Delta}=800 \mathrm{GeV}$ and $\lambda_{1}=10$ are used in plot (b). For clarity, we also show the corresponding $v_{\Delta}$ in the plots (dot-dashed). From the plots, it can be seen that the $H^{++} \rightarrow H^{+} W^{+*}$ decay is the dominant channel when $\lambda_{4}<-0.1(-0.22)$ and $M_{\Delta}=400(800) \mathrm{GeV}$. When $\lambda_{4}>0$, the dominant decay modes are $W^{+} W^{+}$and $\ell_{i}^{+} \ell_{j}^{+}$, where the result with $M_{\Delta}=400 \mathrm{GeV}$ is $B R\left(H^{++} \rightarrow W^{+} W^{+}\right)>B R\left(H^{++} \rightarrow \ell_{i}^{+} \ell_{j}^{+}\right)$; however, the BR order with $M_{\Delta}=800 \mathrm{GeV}$ is reversed due to a smaller $v_{\Delta}$. We note that the relation between $m_{H^{ \pm \pm}}$and $M_{\Delta}\left(\lambda_{1}\right)$ can be written as $m_{H^{ \pm \pm}} \approx \sqrt{M_{\Delta}^{2}+v_{h}^{2} \lambda_{1} / 2}$, which is independent of the $\lambda_{4}$ parameter; therefore, the corresponding $m_{H^{ \pm \pm}}$value can be easily obtained when $M_{\Delta}$ and $\lambda_{1}$ are fixed.

As we discussed in the introduction section, $m_{H^{ \pm \pm}}$lower bound is $770-870 \mathrm{GeV}$ when $H^{ \pm \pm}$dominantly decays into charged leptons. Thus, the scheme with $M_{\Delta}=800 \mathrm{GeV}$ and $\lambda_{1}=10$ has $m_{H^{ \pm \pm}} \approx 971 \mathrm{GeV}$ and can be tested at the LHC. When $H^{ \pm \pm}$predominantly decays into $W^{ \pm} W^{ \pm}$, the lower bound of $m_{H^{ \pm \pm}}$is $\sim 220 \mathrm{GeV}$; therefore, the scheme with $M_{\Delta}=400 \mathrm{GeV}$ and $\lambda_{1}=2.5$, i.e. $M_{H^{ \pm \pm}} \approx 485 \mathrm{GeV}$, is safe from the constraint. 

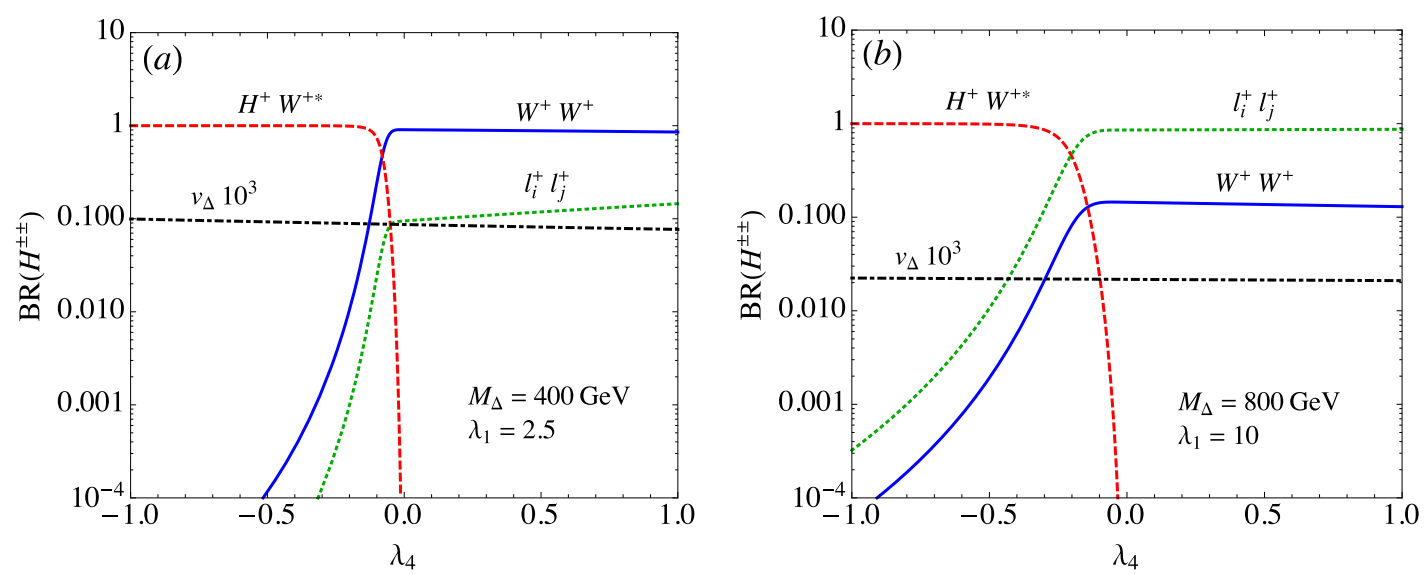

Figure 11. BR of the $H^{++}$decay as a function of $\lambda_{4}$, where (a) $M_{\Delta}=400 \mathrm{GeV}$ and $\lambda_{1}=2.5$ are fixed and (b) $M_{\Delta}=800 \mathrm{GeV}$ and $\lambda_{1}=10$ are used. The dot-dashed line is for $v_{\Delta}$.

\subsection{Singly charged Higgs decays}

In addition to the $\mathrm{H}^{+}$direct couplings to the SM particles, the singly charged Higgs can also decay through mixing with the SM charged-Goldstone boson $\left(G^{+}\right)$, where the relation between the mixing angle $\phi^{+}$and the $v_{\Delta}$ parameter is shown in appendix A. Thus, if the direct $H^{+}$couplings to the SM particles are proportional to $v_{\Delta}$, the mixing effects with $G^{+}$ become important. We find that with the exception of $\ell^{+} \nu$ mode, the decay channels, such as $t \bar{b}, h W^{+}, Z W^{+}$, and $\gamma W^{+}$, are all related to the mixing angle $\phi^{+}$. Hence, the partial decay rates for the fermionic $H^{+}$decays can be expressed as:

$$
\begin{aligned}
\Gamma\left(H^{+} \rightarrow \ell_{i}^{+} \nu\right) & =\frac{m_{H^{ \pm}}}{8 \pi}\left(\mathbf{h}^{\ell \dagger} \mathbf{h}^{\ell}\right)_{i i}, \\
\Gamma\left(H^{+} \rightarrow t \bar{b}\right) & =\frac{m_{H^{ \pm}}}{8 \pi} \frac{m_{t}^{2}}{v_{h}^{2}} s_{\phi^{+}}^{2}\left(1-y_{t}\right)^{2},
\end{aligned}
$$

with $s_{\phi^{+}}\left(c_{\phi^{+}}\right)=\sin \phi^{+}\left(\cos \phi^{+}\right)$and $y_{t}=m_{t}^{2} / m_{H^{ \pm}}^{2}$. Since the $G^{+}$coupling to a quark is proportional to the quark mass [81], we only consider the $t \bar{b}$ mode and the $m_{b}$ effect is neglected due to $m_{b} \ll m_{t}$.

It is found that in addition to the $G^{+} h W^{-}$coupling, $H^{+}$can decay to the $h W^{+}$final state through the mixing between $\operatorname{Re} \Phi$ and $\operatorname{Re} \Delta$, where the mixing effect is dictated by the mixing angle $\alpha$ shown in eq. (A.6). Using the gauge couplings in eq. (B.1) and the $\phi^{+}$and $\alpha$ mixing effects, the partial decay rates for the $H^{+}$diboson decays can then be formulated as:

$$
\begin{aligned}
\Gamma\left(H^{+} \rightarrow h W^{+}\right)= & \frac{g^{2} m_{H^{ \pm}}}{64 \pi}\left(\sqrt{2} s_{\alpha}+s_{\phi^{+}}\right)^{2} \frac{\lambda\left(w_{W}, w_{h}\right)^{3 / 2}}{w_{W}}, \\
\Gamma\left(H^{+} \rightarrow Z W^{+}\right)= & \frac{e^{2} s_{W}^{2} m_{H^{ \pm}}}{16 \pi}\left(\frac{g v_{\Delta}\left(1-3 s_{W}^{2}\right)}{\sqrt{2} m_{W} s_{W}^{2}} c_{\phi^{+}}+s_{\phi^{+}}\right)^{2} \\
& \times w_{Z} \sqrt{\lambda\left(w_{W}, w_{Z}\right)}\left(3+\frac{\lambda\left(w_{W}, w_{Z}\right)}{4 w_{W} w_{Z}}\right), \\
\Gamma\left(H^{+} \rightarrow \gamma W^{+}\right)= & \frac{3 e^{2} m_{H^{ \pm}}}{16 \pi}\left(-\frac{3 g v_{\Delta}}{\sqrt{2} m_{W}} c_{\phi^{+}}+s_{\phi^{+}}\right) w_{W}\left(1-w_{W}\right),
\end{aligned}
$$



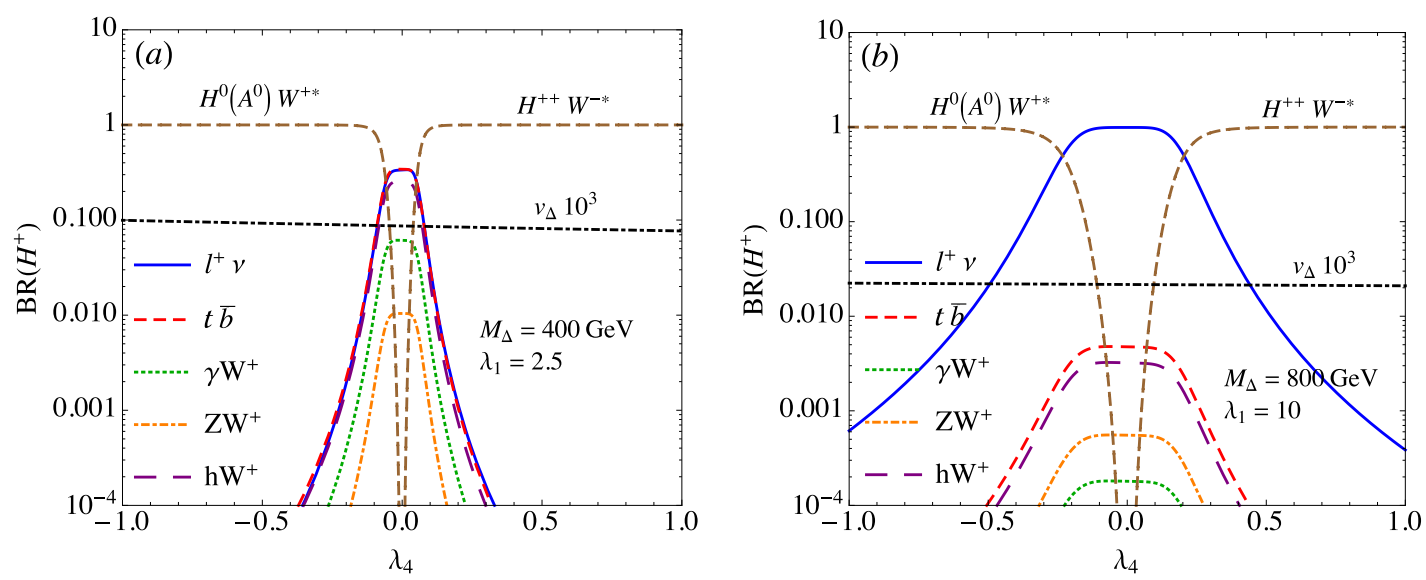

Figure 12. This legend is the same as that shown in figure 11 with the exception of the $H^{+}$decays.

with $w_{i}=m_{i}^{2} / m_{H^{ \pm}}^{2}$. It is known that the $\lambda_{4}$ parameter determines the order of the Higgs triplet masses. Therefore, it is expected that $H^{+}$can decay to $H^{++}$and $H^{0}\left(A^{0}\right)$ through the three-body decay when $\lambda_{4}>0$ and $\lambda_{4}<0$, respectively. Similar to the $H^{++} \rightarrow H^{+} W^{+*}$ decay, we write the partial decay rates for $H^{+} \rightarrow\left(H^{++} W^{-*}, H^{0}\left(A^{0}\right) W^{+*}\right)$ as:

$$
\begin{aligned}
\Gamma\left(H^{+} \rightarrow H^{++} W^{-*}\right) & =\frac{3 g^{4} m_{H^{ \pm}}}{2^{8} \pi^{3}} J_{0}\left(w_{W}, w_{H^{ \pm \pm}}\right), & & \lambda_{4}>0, \\
\Gamma\left(H^{+} \rightarrow S W^{+*}\right) & =\frac{3 g^{4} m_{H^{ \pm}}}{2^{9} \pi^{3}} J_{0}\left(w_{W}, w_{S}\right), & & \lambda_{4}<0,
\end{aligned}
$$

with $S=H^{0}\left(A^{0}\right)$.

Based on the partial decay rate formulations, we show the BR for each decay mode as a function of $\lambda_{4}$ in figure 12(a) and (b), where the plots (a) and (b) correspond to $\left(M_{\Delta}=400 \mathrm{GeV}, \lambda_{1}=2.5\right)$ and $\left(M_{\Delta}=800 \mathrm{GeV}, \lambda_{1}=10\right)$, respectively, and we have summed all possible charged lepton flavors in the $\ell^{+} \nu$ mode. From the plots, it can be clearly seen that when $\left|\lambda_{4}\right|>0.1(0.3)$ for $M_{\Delta}=400(800) \mathrm{GeV}$, the three-body decay channels are the main decays, where the associated mass differences in scalars are $\mid m_{H^{+}}-$ $m_{H^{ \pm \pm}, S} \mid>1.55(2.32) \mathrm{GeV}$. That is, in the model, the two-body $H^{+}$decays can have the significant signals in the scheme with $m_{H^{ \pm \pm}} \approx m_{H^{ \pm}} \approx m_{S}$. In such a degenerate scheme, it is found that for $m_{\Delta}=400 \mathrm{GeV}$, the BRs of the two-body decays follow $B R(\ell \nu) \approx$ $B R(t \bar{b}) \gg B R\left(h W^{+}\right)>B R\left(\gamma W^{+}\right)>B R\left(Z W^{+}\right)$, and for $M_{\Delta}=800 \mathrm{GeV}$, the situation becomes $B R(\ell \nu) \gg B R(t \bar{b})>B R\left(h W^{+}\right) \gg B R\left(Z W^{+}\right)>B R\left(\gamma W^{+}\right)$. For illustration, we show the numerical values with $\lambda_{4}=0$ in table 3 . In addition, in order to understand the scalar mixing influence on the BRs, we show the BRs with $\phi^{+}=\alpha=0$ in figure 13, where $M_{\Delta}=400 \mathrm{GeV}$ and $\lambda_{1}=2.5$ are used. It can be seen that without the $\phi^{+}$and $\alpha$ mixing effects, the contributions to the $t \bar{b}$ and $h W^{+}$modes vanish, and the BR order follows $B R\left(H^{+} \rightarrow \ell^{+} \nu\right)>B R\left(H^{+} \rightarrow Z W^{+}\right)>B R\left(H^{+} \rightarrow \gamma W^{+}\right)$.

\section{$5.4 \quad H^{0}$ and $A^{0}$ decays}

From eq. (3.19), the neutral Higgs triplet scalars do not directly couple to the charged leptons. Thus, without the scalar mixings, the CP-even $H^{0}$ decays to the final states, such 


\begin{tabular}{|c|ccccc|}
\hline Mode & $\ell^{+} \nu$ & $t \bar{b}$ & $h W^{+}$ & $\gamma W^{+}$ & $Z W^{+}$ \\
\hline$\left(M_{\Delta}=400 \mathrm{GeV}, B R\right)$ & 0.34 & 0.34 & 0.25 & 0.06 & 0.01 \\
\hline$\left(M_{\Delta}=800 \mathrm{GeV}, B R\right)$ & 0.99 & 0.005 & 0.003 & $0.17 \cdot 10^{-3}$ & $0.55 \cdot 10^{-3}$ \\
\hline
\end{tabular}

Table 3. BRs of the $H^{+}$decays with $\lambda_{4}=0$, where $\lambda_{1}=2.5$ for $M_{\Delta}=400 \mathrm{GeV}$ and $\lambda_{1}=10$ for $M_{\Delta}=800 \mathrm{GeV}$ are used.

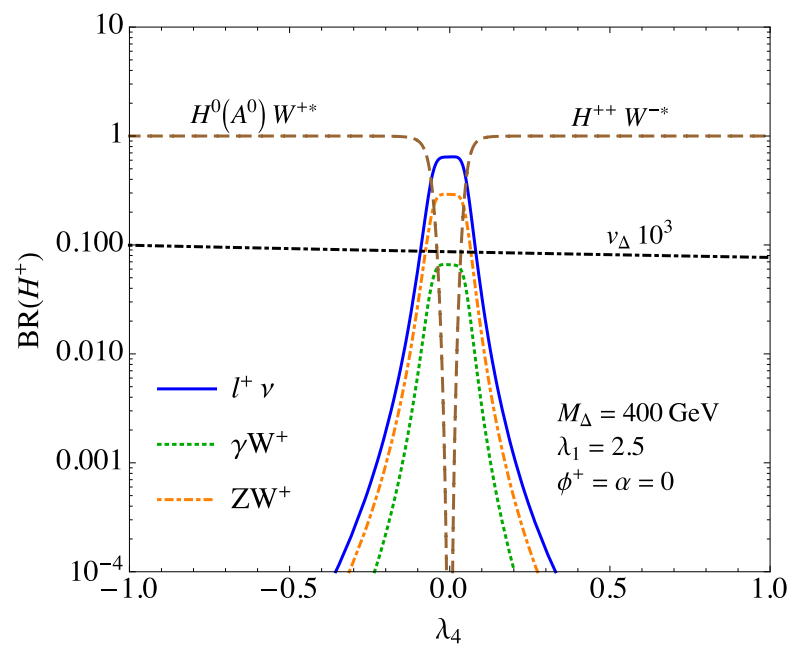

Figure 13. BRs for $H^{+}$decaying to $\ell^{+} \nu, \gamma W^{+}, Z W^{+}, H^{++} W^{-*}$, and $S W^{+*}$, where $\phi^{+}=\alpha=0$, $M_{\Delta}=400 \mathrm{GeV}$, and $\lambda_{1}=2.5$ are used.

as $\nu \nu, h h, W^{+} W^{-}$, and $Z Z$, whereas the CP-odd $A^{0}$ can only has the invisible $A^{0} \rightarrow \nu \nu$ decay. Including the mixings with the SM neutral Goldstone boson $G^{0}$ and with the SM Higgs, it can be found that $H^{0}$ can further decay to $t \bar{t}$ and that $A^{0}$ can decay to $t \bar{t}$ and $h Z$. Therefore, according to the introduced Yukawa and gauge couplings, the partial decay rates of the fermionic $H^{0} / A^{0}$ decays can be expressed as:

$$
\begin{aligned}
\Gamma(S \rightarrow \nu \nu) & =\frac{m_{S}}{8 \pi} \sum_{j}\left(\mathbf{h}^{\ell \dagger} \mathbf{h}^{\ell}\right)_{j j} \\
\Gamma\left(H^{0} \rightarrow t \bar{t}\right) & =\frac{m_{H^{0}}}{8 \pi} \frac{m_{t}^{2}}{v_{h}^{2}} s_{\phi^{0}}^{2}\left(1-\frac{4 m_{t}^{2}}{m_{H^{0}}^{2}}\right)^{3 / 2}, \\
\Gamma\left(A^{0} \rightarrow t \bar{t}\right) & =\frac{m_{A^{0}}}{8 \pi} \frac{m_{t}^{2}}{v_{h}^{2}} s_{\phi^{0}}^{2}\left(1-\frac{4 m_{t}^{2}}{m_{A^{0}}^{2}}\right)^{1 / 2},
\end{aligned}
$$

whereas the $H^{0} / A^{0}$ diboson decays are given as:

$$
\begin{aligned}
\Gamma\left(H^{0} \rightarrow h h\right) & =\frac{m_{H^{0}}}{32 \pi}\left[\left(\lambda_{1}+\lambda_{4}\right) \frac{2 v_{\Delta}-v_{h} s_{\alpha}}{2 m_{H^{0}}}-\sqrt{2} \frac{\mu_{\Delta}}{m_{H^{0}}}\right]^{2}\left(1-\frac{4 m_{h}^{2}}{m_{H^{0}}^{2}}\right)^{1 / 2}, \\
\Gamma\left(H^{0} \rightarrow W^{+} W^{-}\right) & =\frac{g^{2} m_{H^{0}}}{16 \pi}\left(g \frac{v_{\Delta}}{m_{H^{0}}}+\frac{m_{W}}{m_{H^{0}}} s_{\alpha}\right)^{2}\left(2+\frac{\left(1-2 z_{W}\right)^{2}}{4 z_{W}^{2}}\right) \sqrt{1-4 z_{W}}, \\
\Gamma\left(H^{0} \rightarrow Z Z\right) & =\frac{g^{2} m_{H^{0}}}{32 \pi c_{W}^{4}}\left(2 g \frac{v_{\Delta}}{m_{H^{0}}}+\frac{m_{W}}{m_{H^{0}}} s_{\alpha}\right)^{2}\left(2+\frac{\left(1-2 z_{Z}\right)^{2}}{4 z_{Z}^{2}}\right) \sqrt{1-4 z_{Z}}, \\
\Gamma\left(A^{0} \rightarrow h Z\right) & =\frac{g^{2} m_{A^{0}}}{16 \pi}\left(s_{\alpha}+\frac{s_{\phi^{0}}}{2}\right)^{2} \frac{\lambda\left(z_{Z}, z_{h}\right)^{3 / 2}}{z_{W}},
\end{aligned}
$$




\begin{tabular}{|c|ccccc|}
\hline Mode $\left(H^{0}\right)$ & $\nu \nu$ & $t \bar{t}$ & $h h$ & $W^{+} W^{-}$ & $Z Z$ \\
\hline$\left(M_{\Delta}=400 \mathrm{GeV}, B R\right)$ & 0.097 & 0.100 & 0.086 & 0.045 & 0.672 \\
\hline$\left(M_{\Delta}=800 \mathrm{GeV}, B R\right)$ & 0.844 & 0.007 & 0.015 & 0.013 & 0.121 \\
\hline $\operatorname{Mode}\left(A^{0}\right)$ & $\nu \nu$ & $t \bar{t}$ & $h Z$ & & \\
\hline$\left(M_{\Delta}=400 \mathrm{GeV}, B R\right)$ & 0.018 & 0.034 & 0.948 & & \\
\hline$\left(M_{\Delta}=800 \mathrm{GeV}, B R\right)$ & 0.513 & 0.005 & 0.482 & & \\
\hline
\end{tabular}

Table 4. BRs of the $H^{0}$ and $A^{0}$ decays with $\lambda_{4}=0$, where $\lambda_{1}=2.5$ for $M_{\Delta}=400 \mathrm{GeV}$ and $\lambda_{1}=10$ for $M_{\Delta}=800 \mathrm{GeV}$ are used.

with $z_{i}=m_{i}^{2} / m_{S}^{2}$. When $H^{0}\left(A^{0}\right)$ is the heaviest scalar, i.e. $\lambda_{4}>0$, similar to the cases in the $H^{+}$and $H^{++}$decays, the three-body decays $H^{0}\left(A^{0}\right) \rightarrow H^{+} W^{-*}, H^{-} W^{+*}$ are open and the partial decay rates are written as:

$$
\Gamma\left(S \rightarrow H^{+} W^{-*}\right)=\Gamma\left(S \rightarrow H^{-} W^{+*}\right)=\frac{3 g^{4} m_{S}}{2^{9} \pi^{3}} J_{0}\left(z_{W}, z_{H^{ \pm}}\right), \quad \lambda_{4}>0 .
$$

Using the obtained partial decay rates, we show the BR for each decay channel as a function of $\lambda_{4}$ in figure 14, where plots (a) and (b) denote the $H^{0}$ decays with $\left(M_{\Delta}=\right.$ $\left.400 \mathrm{GeV}, \lambda_{1}=2.5\right)$ and $\left(M_{\Delta}=800 \mathrm{GeV}, \lambda_{1}=10\right)$, and plots (c) and (d) are for the $A^{0}$ decays with the same parameter values taken in plots (a) and (b), respectively. From the results, it can be seen that the three-body decays are the dominant decay channels when $\lambda_{4} \gtrsim 0.3$. However, for $\lambda_{4}<0$, the $H^{0}\left(A^{0}\right)$ decay properties depend on the parameter values. For $M_{\Delta}=400 \mathrm{GeV}$ and $\lambda_{1}=2.5$, it can be seen that the BR order in the $H^{0}$ twobody decays follows $B R(Z Z) \gg B R(h h) \sim B R(t \bar{t})>B R(\nu \nu)>B R\left(W^{+} W^{-}\right)$, and that in the $A^{0}$ two-body decays is $B R(h Z) \gg B R(t \bar{t})>B R(\nu \nu)$. For $M_{\Delta}=800 \mathrm{GeV}$ and $\lambda_{1}=10$, the $\mathrm{BR}$ order in the $H^{0}$ decays is $B R(\nu \bar{\nu}) \gg B R(Z Z)>B R(h h)>B R\left(W^{+} W^{-}\right)>$ $B R(t \bar{t})$, and that in the $A^{0}$ decays is $B R(h Z) \sim B R(\nu \bar{\nu}) \gg B R(t \bar{t})$. For clarity, we show the numerical values for the $H^{0}$ and $A^{0}$ decays with $\lambda_{4}=0$ in table 4 . In order to illustrate the $\phi^{0}$ and $\alpha$ mixing angle influence, we show the relative BRs as a function of $\lambda_{4}$ with $\phi^{0}=\alpha=0$ in figure 15 , where $m_{\Delta}=400 \mathrm{GeV}$ and $\lambda_{1}=2.5$ are fixed. According to the results, it can be found that $B R\left(H^{0} \rightarrow t \bar{t}\right)$ vanishes and that $B R\left(H^{0} \rightarrow W^{+} W^{-}\right) \sim 0.3$, which is close to $B R\left(H^{0} \rightarrow Z Z\right)$. Accordingly, we see that the $\mathrm{BR}$ of $H^{0} \rightarrow W^{+} W^{-}$ obtains a destructive contribution from the $\alpha$ mixing effect. When $\phi^{0}=\alpha=0, A^{0}$ only can decay to $\nu \nu$ in the region of $\lambda_{4}<0$; therefore, we do not explicitly show the situation for the $A^{0}$ decay.

\section{Conclusion}

Using the scotogenic approach, we studied the radiatively induced lepton-number violation dimension-3 term $\mu_{\Delta} H^{T} i \tau_{2} \Delta^{\dagger} H$ in the base of the type-II seesaw model, where the introduced dark vector-like doublet lepton $X$ and dark right-handed singlet Majorana lepton $N$ are the mediators in the loop. It was found that the dynamically induced Higgs triplet VEV is limited in the region of $10^{-5}-10^{-4} \mathrm{GeV}$ when the relevant parameters satisfy the constraints from the DM measurements. Due to the DM direct detection constraints, only 

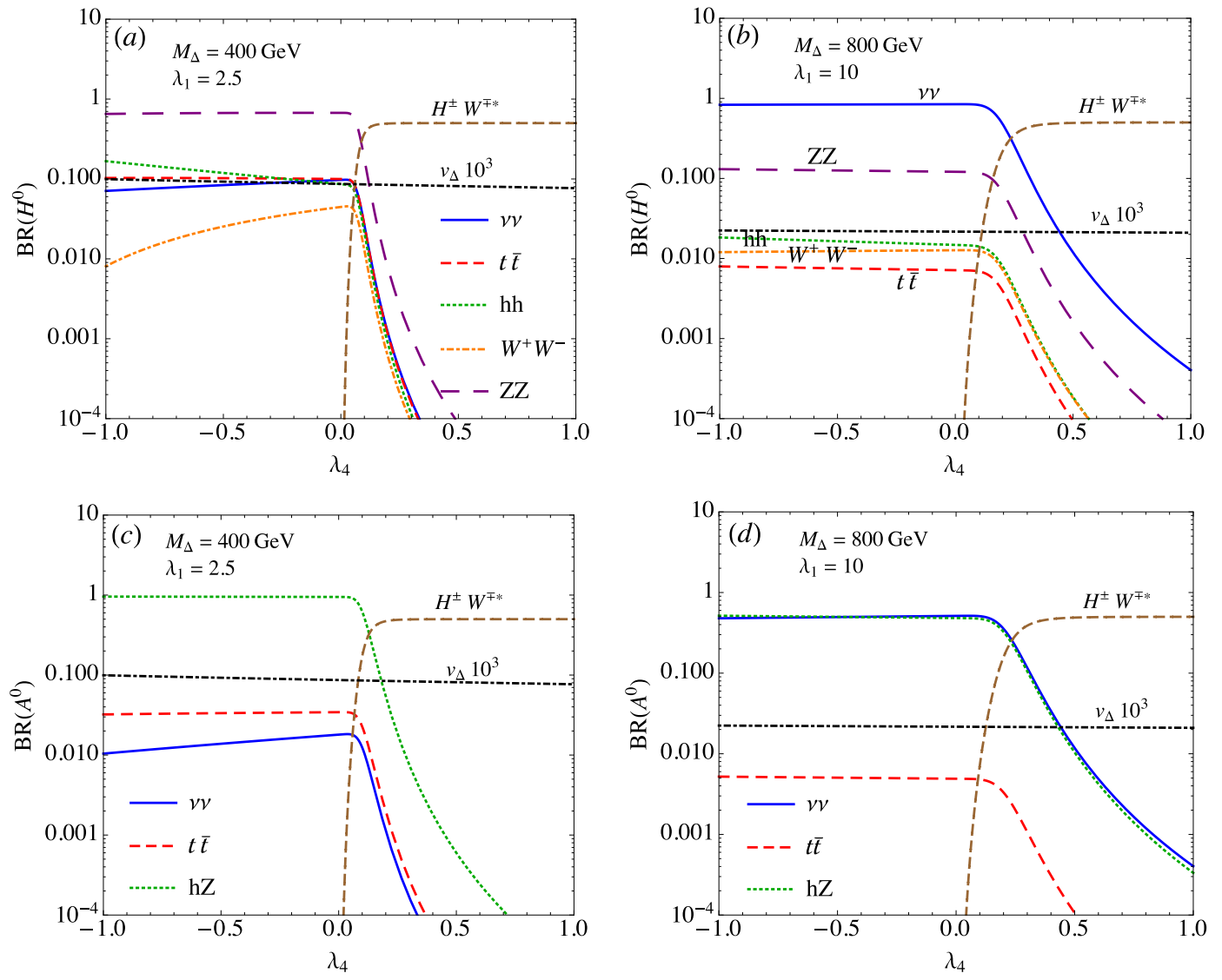

Figure 14. This legend is the same as that shown in figure 11, where plots (a) and (b) are for $H^{0}$ decays, and plots (c) and (d) are for $A^{0}$ decays.

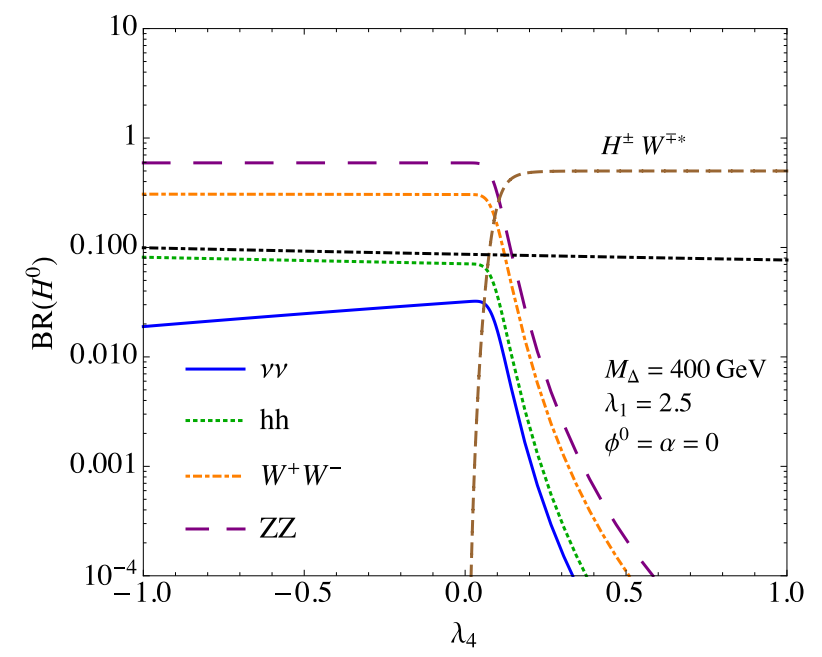

Figure 15. BRs for $H^{0}$ decay into $\nu \nu, h h, W^{+} W^{-}, Z Z$, and $H^{ \pm} W^{\mp *}$, where $\phi^{+}=\alpha=0$, $M_{\Delta}=400 \mathrm{GeV}$, and $\lambda_{1}=2.5$ are used. 
the singlet Majorana lepton can be the DM candidate in the model, and the DM mass depends on and is close to the $m_{X}$ parameter.

In the model, the Higgs triplet VEV, $v_{\Delta}$, depends not only on the $\mu_{\Delta}$ and $M_{\Delta}$ parameters, but also on the $\lambda_{1,4}$ parameters in the scalar potential, which dictate the SM Higgs couplings to the doubly and singly charged Higgses. Moreover, the mass ordering of the Higgs triplet scalars is dictated by the $\lambda_{4}$ sign. We showed that the Higgs diphoton decay and the oblique $T$-parameter can further bound the $\lambda_{1,4}$ parameters. As a result, we obtain $\left|m_{H^{ \pm \pm}}-m_{H^{ \pm}}\right| \lesssim 50 \mathrm{GeV}$.

We did not explicitly study the collider signatures in this work. Rather, we analyzed the decay channels of each Higgs triplet scalar and estimated the associated branching ratios in detail. We found that the scalar mixing effects have an important influence on the partial decay rates of the singly charged-Higgs, CP-even scalar, and CP-odd pseudoscalar in the near degenerate masses (i.e. $\lambda_{4} \ll 1$ ). In the non-degenerate mass region, the branching ratios of the Higgs triplet scalar decays are dominated by the three-body decays when they are kinematically allowed.

\section{A Scalar mass squares and mixing angles}

The symmetric mass-square matrices in eqs. (3.9), (3.10), and (3.11) can be generally expressed as:

$$
A=\left(\begin{array}{ll}
a_{11} & a_{12} \\
a_{12} & a_{22}
\end{array}\right),
$$

where the $2 \times 2$ symmetric matrix can be diagonalized using an orthogonal matrix $U$ through $A^{\text {dia }}=U A U^{T}$ with the parametrization:

$$
U=\left(\begin{array}{cc}
\cos \phi & -\sin \phi \\
\sin \phi & \cos \phi
\end{array}\right) .
$$

It can be found that the two eigenvalues $A_{L}$ and $A_{H}$ and the mixing angle $\phi$ can be expressed as:

$$
\begin{aligned}
A_{L(H)} & =\frac{a_{11}+a_{22}}{2} \mp \frac{1}{2} \sqrt{\left(a_{11}-a_{22}\right)^{2}+4 a_{12}^{2}}, \\
\tan 2 \phi & =\frac{2 a_{12}}{a_{22}-a_{11}} .
\end{aligned}
$$

Since the $\left(G^{+}, \Delta^{+}\right)$and $\left(G^{0}, \operatorname{Im} \Delta^{0}\right)$ states have massless Goldstone bosons, their physical mass squares can be straightforwardly obtained by taking traces of the mass-square matrices, i.e. $m_{H^{+}}^{2}=\operatorname{Tr} A_{G^{+} \Delta^{+}}$and $m_{A^{0}}^{2}=\operatorname{Tr} A_{G^{0} \operatorname{Im} \Delta^{0}}$. From eq. (A.3), the corresponding mixing angles for diagonalizing $A_{G^{+} \Delta^{+}}$and $A_{G^{0} \mathrm{Im} \Delta^{0}}$ shown in eqs. (3.9) and (3.10) are given as:

$$
\begin{aligned}
\tan 2 \phi^{+} & =\frac{-2 \sqrt{2} v_{\Delta} v_{h}}{v_{h}^{2}-2 v_{\Delta}^{2}} \approx-\frac{2 \sqrt{2} v_{\Delta}}{v_{h}}, \\
\tan 2 \phi^{0} & =\frac{-4 v_{\Delta} v_{h}}{v_{h}^{2}-4 v_{\Delta}^{2}} \approx-\frac{4 v_{\Delta}}{v_{h}} .
\end{aligned}
$$


Clearly, if $v_{\Delta} \ll v_{h}$, the mixing angles are small. In the case of the $\left(\operatorname{Re} \Phi^{0}, \operatorname{Re} \Delta^{0}\right)$ states, we do not have a simple way to obtain their eigenvalues. If we use $h$ and $H^{0}$ to denote the light and heavy scalars, their eigenvalues $m_{h\left(H^{0}\right)}$ and mixing angles should follow eq. (A.3), where the associated matrix elements are:

$$
\begin{aligned}
& a_{11}=\frac{\lambda v_{h}^{2}}{2}, \\
& a_{12}=\left(\lambda_{1}+\lambda_{4}\right) v_{h} v_{\Delta}-\sqrt{2} v_{h} \mu_{\Delta}, \\
& a_{22}=\frac{\mu_{\Delta} v_{h}^{2}}{\sqrt{2} v_{\Delta}}+2 v_{\Delta}^{2}\left(\lambda_{2}+\lambda_{3}\right) .
\end{aligned}
$$

As a result, the mixing between $\operatorname{Re} \Phi^{0}$ and $\operatorname{Re} \Delta^{0}$ can be formulated as:

$$
\tan 2 \alpha \approx \frac{2\left(\lambda_{1}+\lambda_{4}\right) v_{\Delta}-2 \sqrt{2} \mu_{\Delta}}{\mu_{\Delta} v_{h} /\left(\sqrt{2} v_{\Delta}\right)-\lambda v_{h}^{2} / 2}
$$

where we have used $\alpha$ instead of $\phi$, and the $v_{\Delta}^{2}$ effect in the denominator is dropped due to $v_{\Delta}^{2} \ll 1$. In addition to $v_{\Delta}<\mu_{\Delta}$, the numerator in eq. (A.6) is much smaller than the denominator; hence, the $\alpha$ angle should be of the order of $\sim \mu_{\Delta} v_{h} / M_{\Delta}^{2}$. Using $\mu_{\Delta}=10^{-3} \mathrm{GeV}, v_{h}=246 \mathrm{GeV}$, and $M_{\Delta}=400 \mathrm{GeV}$, the $\alpha$ value can be estimated to be $\alpha \sim 1.54 \times 10^{-6}$.

\section{B Higgs triplet gauge coupling}

The Higgs triplet couplings to the gauge bosons can be obtained from the $\Delta$ kinetic term shown in eq. (3.15), where the covariant derivation can be found in eq. (3.16). Accordingly, we can derive the triple couplings of the Higgs triplet scalars and the gauge bosons as:

$$
\begin{aligned}
\mathcal{L}_{\text {kin }}= & \operatorname{Tr}\left[\left(D_{\mu} \Delta\right)^{\dagger}\left(D^{\mu} \Delta\right)\right] \\
\supset & \left\{i g\left(H^{--} \partial_{\mu} H^{+}-H^{+} \partial_{\mu} H^{--}\right) W^{+\mu}+\frac{i g}{\sqrt{2}}\left(H^{0} \partial_{\mu} H^{-1}-H^{-1} \partial_{\mu} H^{0}\right) W^{+\mu}\right. \\
& \left.-\frac{g}{\sqrt{2}}\left(A^{0} \partial_{\mu} H^{-1}-H^{-1} \partial_{\mu} A^{0}\right) W^{+\mu}+\text { H.c. }\right\}-\frac{g}{c_{W}}\left(H^{0} \partial_{\mu} A^{0}-A^{0} \partial_{\mu} H^{0}\right) Z^{\mu} \\
& +i\left(H^{+} \partial_{\mu} H^{-}-H^{-} \partial_{\mu} H^{+}\right)\left(e A_{\mu}-\frac{g s_{W}^{2}}{c_{W}} Z_{\mu}\right) \\
& +i\left(H^{++} \partial_{\mu} H^{--}-H^{--} \partial_{\mu} H^{++}\right)\left(2 e A_{\mu}+\frac{g\left(1-2 s_{W}^{2}\right)}{c_{W}} Z_{\mu}\right) \\
& +g^{2} v_{\Delta} H^{0} W_{\mu}^{+} W^{-\mu}+\frac{1}{2}\left(\frac{2 g^{2} v_{\Delta}}{c_{W}^{2}}\right) H^{0} Z_{\mu} Z^{\mu} \\
& -\frac{g v_{\Delta}}{\sqrt{2}}\left[H^{-} W^{+\mu}\left(3 e A_{\mu}+\frac{g}{c_{W}}\left(1-3 s_{W}^{2}\right) Z_{\mu}\right)+\text { H.c. }\right] \\
& -\frac{1}{2}\left(\sqrt{2} g^{2} v_{\Delta}\right)\left(H^{--} W_{\mu}^{+} W^{+\mu}+\text { H.c. }\right) .
\end{aligned}
$$

We note that although eq. (B.1) does not include the $\phi^{+, 0}$ and $\alpha$ mixing effects, we have used the physical state notations for $H^{+}, H^{0}$, and $A^{0}$. 


\section{Loop integral functions}

The loop integral functions $A_{0,1 / 2,1}^{h}$ for $h \rightarrow Z \gamma$ shown in eq. (5.3) are given as:

$$
\begin{aligned}
& A_{0}^{h}\left(\tau_{h}, \tau_{Z}\right)=I_{1}\left(\tau_{h}, \tau_{Z}\right), A_{1 / 2}^{h}=I_{1}\left(\tau_{h}, \tau_{Z}\right)-I_{2}\left(\tau_{h}, \tau_{Z}\right), \\
& A_{1}^{h}\left(\tau_{h}, \tau_{Z}\right)=4\left(3-\tan ^{2} \theta_{W}\right) I_{2}\left(\tau_{h}, \tau_{Z}\right)+\left[\left(1+\frac{2}{\tau_{h}}\right) \tan _{W}^{2}-\left(5+\frac{2}{\tau_{h}}\right)\right] I_{1}\left(\tau_{h}, \tau_{Z}\right),
\end{aligned}
$$

with

$$
\begin{aligned}
& I_{1}(x, y)=\frac{x y}{2(x-y)}+\frac{x^{2} y^{2}}{2(x-y)^{2}}(f(x)-f(y))+\frac{x^{2} y}{(x-y)^{2}}(g(x)-g(y)), \\
& I_{2}(x, y)=-\frac{x y}{2(x-y)}(f(x)-f(y)),
\end{aligned}
$$

where the function $f(\tau)$ can be found in eq. (4.14), and the function $g(\tau)$ is given as:

$$
g(\tau)= \begin{cases}\sqrt{\tau-1} \sin ^{-1}(1 / \sqrt{\tau}), & (\tau \geq 1), \\ \frac{\sqrt{1-\tau}}{2}\left(\ln \frac{1+\sqrt{1-\tau}}{1-\sqrt{1-\tau}}-i \pi\right), & (\tau<1) .\end{cases}
$$

\section{Acknowledgments}

This work was partially supported by the Ministry of Science and Technology of Taiwan, under grants MOST-106-2112-M-006-010-MY2 (CHC).

Open Access. This article is distributed under the terms of the Creative Commons Attribution License (CC-BY 4.0), which permits any use, distribution and reproduction in any medium, provided the original author(s) and source are credited.

\section{References}

[1] F. Englert and R. Brout, Broken symmetry and the mass of gauge vector mesons, Phys. Rev. Lett. 13 (1964) 321 [INSPIRE].

[2] P.W. Higgs, Broken symmetries and the masses of gauge bosons, Phys. Rev. Lett. 13 (1964) 508 [INSPIRE].

[3] G.S. Guralnik, C.R. Hagen and T.W.B. Kibble, Global conservation laws and massless particles, Phys. Rev. Lett. 13 (1964) 585 [INSPIRE].

[4] J. Schechter and J.W.F. Valle, Neutrino masses in $\mathrm{SU}(2) \times \mathrm{U}(1)$ theories, Phys. Rev. D 22 (1980) 2227 [INSPIRE].

[5] M. Magg and C. Wetterich, Neutrino mass problem and gauge hierarchy, Phys. Lett. B 94 (1980) 61.

[6] T.P. Cheng and L.-F. Li, Neutrino masses, mixings and oscillations in $\mathrm{SU}(2) \times \mathrm{U}(1)$ models of electroweak interactions, Phys. Rev. D 22 (1980) 2860 [InSPIRE].

[7] G. Lazarides, Q. Shafi and C. Wetterich, Proton lifetime and fermion masses in an $\mathrm{SO}(10)$ model, Nucl. Phys. B 181 (1981) 287 [INSPIRE]. 
[8] R.N. Mohapatra and G. Senjanović, Neutrino masses and mixings in gauge models with spontaneous parity violation, Phys. Rev. D 23 (1981) 165 [InSPIRE].

[9] E.J. Chun, K.Y. Lee and S.C. Park, Testing Higgs triplet model and neutrino mass patterns, Phys. Lett. B 566 (2003) 142 [hep-ph/0304069] [INSPIRE].

[10] R. Franceschini and R.N. Mohapatra, Radiatively induced type-II seesaw models and vectorlike 5/3 charge quarks, Phys. Rev. D 89 (2014) 055013 [arXiv: 1306.6108] [INSPIRE].

[11] Y. Cai et al., From the trees to the forest: a review of radiative neutrino mass models, Front. in Phys. 5 (2017) 63 [arXiv:1706. 08524] [inSPIRE].

[12] E. Ma, Verifiable radiative seesaw mechanism of neutrino mass and dark matter, Phys. Rev. D 73 (2006) 077301 [hep-ph/0601225] [INSPIRE].

[13] S. Fraser, C. Kownacki, E. Ma and O. Popov, Type II radiative Seesaw model of neutrino mass with dark matter, Phys. Rev. D 93 (2016) 013021 [arXiv:1511.06375] [INSPIRE].

[14] V. Brdar, I. Picek and B. Radovcic, Radiative neutrino mass with scotogenic scalar triplet, Phys. Lett. B 728 (2014) 198 [arXiv:1310.3183] [INSPIRE].

[15] E. Ma, Vanishing Higgs one-loop quadratic divergence in the scotogenic model and beyond, Phys. Lett. B 732 (2014) 167 [arXiv:1401.3284] [INSPIRE].

[16] E. Molinaro, C.E. Yaguna and O. Zapata, FIMP realization of the scotogenic model, JCAP 07 (2014) 015 [arXiv: 1405.1259] [INSPIRE].

[17] A. Vicente and C.E. Yaguna, Probing the scotogenic model with lepton flavor violating processes, JHEP 02 (2015) 144 [arXiv: 1412.2545] [INSPIRE].

[18] A. Merle and M. Platscher, Parity problem of the scotogenic neutrino model, Phys. Rev. D 92 (2015) 095002 [arXiv: 1502.03098] [INSPIRE].

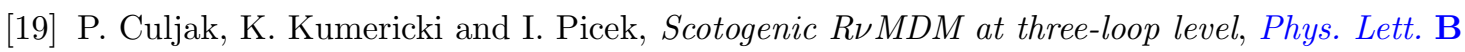
744 (2015) 237 [arXiv: 1502.07887] [INSPIRE].

[20] A. Merle and M. Platscher, Running of radiative neutrino masses: the scotogenic model Revisited, JHEP 11 (2015) 148 [arXiv:1507.06314] [INSPIRE].

[21] J.-H. Yu, Hidden gauged U(1) model: unifying scotogenic neutrino and flavor dark matter, Phys. Rev. D 93 (2016) 113007 [arXiv: 1601.02609] [INSPIRE].

[22] A. Ahriche, K.L. McDonald and S. Nasri, The scale-invariant scotogenic model, JHEP 06 (2016) 182 [arXiv: 1604.05569] [INSPIRE].

[23] P.M. Ferreira, W. Grimus, D. Jurciukonis and L. Lavoura, Scotogenic model for co-bimaximal mixing, JHEP 07 (2016) 010 [arXiv: 1604.07777] [INSPIRE].

[24] P. Rocha-Moran and A. Vicente, Lepton flavor violation in the singlet-triplet scotogenic model, JHEP 07 (2016) 078 [arXiv: 1605.01915] [INSPIRE].

[25] T.A. Chowdhury and S. Nasri, The Sommerfeld enhancement in the scotogenic model with large electroweak scalar multiplets, JCAP 01 (2017) 041 [arXiv: 1611.06590] [INSPIRE].

[26] A.G. Hessler, A. Ibarra, E. Molinaro and S. Vogl, Probing the scotogenic FIMP at the LHC, JHEP 01 (2017) 100 [arXiv: 1611.09540] [INSPIRE].

[27] M.A. Díaz, N. Rojas, S. Urrutia-Quiroga and J.W.F. Valle, Heavy Higgs boson production at colliders in the singlet-triplet scotogenic dark matter model, JHEP 08 (2017) 017 [arXiv: 1612.06569] [INSPIRE].

[28] D. Borah and A. Gupta, New viable region of an inert Higgs doublet dark matter model with scotogenic extension, Phys. Rev. D 96 (2017) 115012 [arXiv:1706.05034] [INSPIRE]. 
[29] A. Abada and T. Toma, Electric dipole moments in the minimal scotogenic model, JHEP 04 (2018) 030 [arXiv : 1802.00007] [INSPIRE].

[30] C. Hagedorn, J. Herrero-García, E. Molinaro and M.A. Schmidt, Phenomenology of the generalised scotogenic model with fermionic dark matter, JHEP 11 (2018) 103 [arXiv: 1804.04117] [INSPIRE].

[31] T. Hugle, M. Platscher and K. Schmitz, Low-Scale leptogenesis in the scotogenic neutrino mass model, Phys. Rev. D 98 (2018) 023020 [arXiv:1804.09660] [INSPIRE].

[32] S. Baumholzer, V. Brdar and P. Schwaller, The new $\nu M S M$ ( $\nu \nu M S M)$ : radiative neutrino masses, keV-scale dark matter and viable leptogenesis with sub-TeV new physics, JHEP 08 (2018) 067 [arXiv: 1806.06864] [INSPIRE].

[33] N. Rojas, R. Srivastava and J.W.F. Valle, Simplest scoto-seesaw mechanism, Phys. Lett. B 789 (2019) 132 [arXiv: 1807.11447] [INSPIRE].

[34] D. Borah, P.S.B. Dev and A. Kumar, TeV scale leptogenesis, inflaton dark matter and neutrino mass in a scotogenic model, Phys. Rev. D 99 (2019) 055012 [arXiv:1810.03645] [INSPIRE].

[35] S. Centelles Chuliá, R. Cepedello, E. Peinado and R. Srivastava, Scotogenic dark symmetry as a residual subgroup of standard model symmetries, arXiv:1901.06402 [INSPIRE].

[36] E. Ma, Scotogenic U(1) \ Dirac neutrinos, Phys. Lett. B 793 (2019) 411 [arXiv:1901.09091] [INSPIRE].

[37] S.K. Kang et al., Scotogenic dark matter stability from gauged matter parity, arXiv: 1902.05966 [INSPIRE].

[38] C.-H. Chen and T. Nomura, Influence of an inert charged Higgs boson on the muon $g-2$ and radiative neutrino masses in a scotogenic model, Phys. Rev. D 100 (2019) 015024 [arXiv: 1903.03380] [INSPIRE].

[39] S. Kanemura and H. Sugiyama, Dark matter and a suppression mechanism for neutrino masses in the Higgs triplet model, Phys. Rev. D 86 (2012) 073006 [arXiv:1202.5231] [INSPIRE].

[40] T. Nomura, H. Okada and Y. Orikasa, Radiative neutrino model with $\mathrm{SU}(2)_{L}$ triplet fields, Phys. Rev. D 94 (2016) 115018 [arXiv:1610.04729] [INSPIRE].

[41] T. Nomura and H. Okada, Loop induced type-II seesaw model and GeV dark matter with $\mathrm{U}(1)_{B-L}$ gauge symmetry, Phys. Lett. B 774 (2017) 575 [arXiv: 1704.08581] [INSPIRE].

[42] M.E. Peskin and T. Takeuchi, Estimation of oblique electroweak corrections, Phys. Rev. D 46 (1992) 381 [INSPIRE].

[43] CMS collaboration, A search for doubly-charged Higgs boson production in three and four lepton final states at $\sqrt{s}=13 \mathrm{TeV}$, CMS-PAS-HIG-16-036 (2016).

[44] ATLAS collaboration, Search for doubly charged Higgs boson production in multi-lepton final states with the ATLAS detector using proton-proton collisions at $\sqrt{s}=13 \mathrm{TeV}$, Eur. Phys. J. C 78 (2018) 199 [arXiv:1710.09748] [INSPIRE].

[45] ATLAS collaboration, Search for doubly charged scalar bosons decaying into same-sign $W$ boson pairs with the ATLAS detector, Eur. Phys. J. C 79 (2019) 58 [arXiv:1808.01899] [INSPIRE].

[46] ATLAS collabroation, Searches for doubly charged Higgs bosons with the ATLAS detector, PoS (CHARGED 2018) 008. 
[47] A.G. Akeroyd and M. Aoki, Single and pair production of doubly charged Higgs bosons at hadron colliders, Phys. Rev. D 72 (2005) 035011 [hep-ph/0506176] [INSPIRE].

[48] F. del Aguila and J.A. Aguilar-Saavedra, Distinguishing seesaw models at LHC with multi-lepton signals, Nucl. Phys. B 813 (2009) 22 [arXiv:0808.2468] [INSPIRE].

[49] A. Melfo et al., Type II seesaw at LHC: the roadmap, Phys. Rev. D 85 (2012) 055018 [arXiv: 1108.4416] [INSPIRE].

[50] M. Aoki, S. Kanemura and K. Yagyu, Testing the Higgs triplet model with the mass difference at the LHC, Phys. Rev. D 85 (2012) 055007 [arXiv:1110.4625] [inSPIRE].

[51] A.G. Akeroyd and H. Sugiyama, Production of doubly charged scalars from the decay of singly charged scalars in the Higgs triplet model, Phys. Rev. D 84 (2011) 035010 [arXiv:1105.2209] [INSPIRE].

[52] A. Arhrib et al., Higgs boson decay into 2 photons in the type II Seesaw Model, JHEP 04 (2012) 136 [arXiv:1112.5453] [INSPIRE].

[53] A.G. Akeroyd, S. Moretti and H. Sugiyama, Five-lepton and six-lepton signatures from production of neutral triplet scalars in the Higgs Triplet Model, Phys. Rev. D 85 (2012) 055026 [arXiv: 1201.5047 ] [INSPIRE].

[54] C.-W. Chiang, T. Nomura and K. Tsumura, Search for doubly charged Higgs bosons using the same-sign diboson mode at the LHC, Phys. Rev. D 85 (2012) 095023 [arXiv:1202.2014] [INSPIRE].

[55] E.J. Chun and P. Sharma, Same-sign tetra-leptons from type II seesaw, JHEP 08 (2012) 162 [arXiv:1206.6278] [INSPIRE].

[56] E.J. Chun and P. Sharma, Search for a doubly-charged boson in four lepton final states in type-II seesaw, Phys. Lett. B 728 (2014) 256 [arXiv:1309.6888] [INSPIRE].

[57] M. Chabab, M.C. Peyranere and L. Rahili, Degenerate Higgs bosons decays to $\gamma \gamma$ and $Z \gamma$ in the type-II seesaw model, Phys. Rev. D 90 (2014) 035026 [arXiv:1407.1797] [INSPIRE].

[58] Z.-L. Han, R. Ding and Y. Liao, LHC phenomenology of type II seesaw: nondegenerate case, Phys. Rev. D 91 (2015) 093006 [arXiv: 1502.05242] [INSPIRE].

[59] S.-Y. Guo, Z.-L. Han and Y. Liao, Testing the type-II radiative seesaw model: from dark matter detection to LHC signatures, Phys. Rev. D 94 (2016) 115014 [arXiv:1609.01018] [INSPIRE].

[60] M. Mitra, S. Niyogi and M. Spannowsky, Type-II seesaw model and multilepton signatures at hadron colliders, Phys. Rev. D 95 (2017) 035042 [arXiv:1611.09594] [INSPIRE].

[61] D.K. Ghosh, N. Ghosh, I. Saha and A. Shaw, Revisiting the high-scale validity of the type-II seesaw model with novel LHC signature, Phys. Rev. D 97 (2018) 115022 [arXiv: 1711.06062] [INSPIRE].

[62] P.S.B. Dev, M.J. Ramsey-Musolf and Y. Zhang, Doubly-charged scalars in the type-II seesaw mechanism: fundamental symmetry tests and high-energy searches, Phys. Rev. D 98 (2018) 055013 [arXiv: 1806. 08499] [INSPIRE].

[63] P.S. Bhupal Dev and Y. Zhang, Displaced vertex signatures of doubly charged scalars in the type-II seesaw and its left-right extensions, JHEP 10 (2018) 199 [arXiv:1808.00943] [INSPIRE].

[64] Y. Du, A. Dunbrack, M.J. Ramsey-Musolf and J.-H. Yu, Type-II seesaw scalar triplet model at a 100 TeV pp collider: discovery and Higgs portal coupling determination, JHEP 01 (2019) 101 [arXiv: 1810.09450] [INSPIRE]. 
[65] S. Antusch, O. Fischer, A. Hammad and C. Scherb, Low scale type-II seesaw: Present constraints and prospects for displaced vertex searches, JHEP 02 (2019) 157 [arXiv: 1811.03476] [INSPIRE].

[66] S. Bhattacharya, P. Ghosh, N. Sahoo and N. Sahu, Mini review on vector-like leptonic dark matter, neutrino mass and collider signatures, Front. in Phys. 7 (2019) 80 [arXiv: 1812.06505] [INSPIRE].

[67] B. Barman et al., Fermion dark matter with scalar triplet at direct and collider searches, Phys. Rev. D 100 (2019) 015027 [arXiv:1902.01217] [INSPIRE].

[68] R. Primulando, J. Julio and P. Uttayarat, Scalar phenomenology in type-II seesaw model, JHEP 08 (2019) 024 [arXiv: 1903.02493] [INSPIRE].

[69] XENON collaboration, Dark matter search results from a one ton-year exposure of XENON1T, Phys. Rev. Lett. 121 (2018) 111302 [arXiv:1805.12562] [INSPIRE].

[70] PICO collaboration, Dark matter search results from the PICO-60 $C_{3} F_{8}$ bubble chamber, Phys. Rev. Lett. 118 (2017) 251301 [arXiv:1702.07666] [INSPIRE].

[71] XENON collaboration, Constraining the spin-dependent WIMP-nucleon cross sections with XENON1T, Phys. Rev. Lett. 122 (2019) 141301 [arXiv:1902.03234] [inSPIRE].

[72] C. Bonilla, R.M. Fonseca and J.W.F. Valle, Consistency of the triplet seesaw model revisited, Phys. Rev. D 92 (2015) 075028 [arXiv: 1508. 02323] [INSPIRE].

[73] G. Arcadi, A. Djouadi and M. Raidal, Dark matter through the Higgs portal, arXiv: 1903.03616 [INSPIRE].

[74] A. Alves, A. Berlin, S. Profumo and F.S. Queiroz, Dark matter complementarity and the $Z^{\prime}$ portal, Phys. Rev. D 92 (2015) 083004 [arXiv:1501.03490] [INSPIRE].

[75] G. Bélanger, F. Boudjema, A. Pukhov and A. Semenov, Dark matter direct detection rate in a generic model with MicrOMEGAs 2.2, Comput. Phys. Commun. 180 (2009) 747 [arXiv:0803.2360] [INSPIRE].

[76] L. Lavoura and L.-F. Li, Making the small oblique parameters large, Phys. Rev. D 49 (1994) 1409 [hep-ph/9309262] [INSPIRE].

[77] A. Arhrib et al., The Higgs potential in the type II seesaw model, Phys. Rev. D 84 (2011) 095005 [arXiv: 1105.1925] [INSPIRE].

[78] K. Kannike, Vacuum stability conditions from copositivity criteria, Eur. Phys. J. C 72 (2012) 2093 [arXiv: 1205.3781] [INSPIRE].

[79] Particle Data Group collaboration, Review of particle physics, Phys. Rev. D 98 (2018) 030001 [INSPIRE].

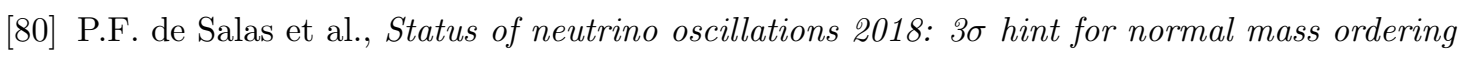
and improved CP sensitivity, Phys. Lett. B 782 (2018) 633 [arXiv:1708.01186] [InSPIRE].

[81] J.F. Gunion, H.E. Haber, G.L. Kane and S. Dawson, Errata for the Higgs hunter's guide, hep-ph/9302272 [INSPIRE].

[82] A. Denner et al., Standard model Higgs-boson branching ratios with uncertainties, Eur. Phys. J. C 71 (2011) 1753 [arXiv:1107.5909] [INSPIRE].

[83] ATLAS collaboration, Combined measurements of Higgs boson production and decay using up to $80 \mathrm{fb}^{-1}$ of proton-proton collision data at $\sqrt{s}=13$ TeV collected with the ATLAS experiment, ATLAS-CONF-2019-005 (2019).

[84] CMS collaboration, Measurements of Higgs boson production via gluon fusion and vector boson fusion in the diphoton decay channel at $\sqrt{s}=13 \mathrm{TeV}$, CMS-PAS-HIG-18-029 (2018). 
[85] E.J. Chun, H.M. Lee and P. Sharma, Vacuum stability, perturbativity, EWPD and Higgs-to-diphoton rate in type II seesaw models, JHEP 11 (2012) 106 [arXiv:1209.1303] [INSPIRE].

[86] Planck collaboration, Planck 2015 results. XIII. Cosmological parameters, Astron. Astrophys. 594 (2016) A13 [arXiv:1502.01589] [INSPIRE].

[87] R.N. Cahn, M.S. Chanowitz and N. Fleishon, Higgs particle production by $Z \rightarrow H \gamma$, Phys. Lett. B 82 (1979) 113.

[88] L. Bergstrom and G. Hulth, Induced Higgs couplings to neutral bosons in $e^{+} e^{-}$collisions, Nucl. Phys. B 259 (1985) 137 [Erratum ibid. B 276 (1986) 744] [InSPIRE].

[89] F. Arbabifar, S. Bahrami and M. Frank, Neutral Higgs bosons in the Higgs triplet model with nontrivial mixing, Phys. Rev. D 87 (2013) 015020 [arXiv:1211.6797] [INSPIRE].

[90] P.S. Bhupal Dev, D.K. Ghosh, N. Okada and I. Saha, 125 GeV Higgs boson and the type-II seesaw model, JHEP 03 (2013) 150 [Erratum ibid. 05 (2013) 049] [arXiv:1301.3453] [INSPIRE]. 